\title{
Semi-analytical static analysis of nonlocal strain gradient laminated composite nanoplates in hygrothermal environment
}

\author{
Giovanni Tocci Monaco $^{1,2} \cdot$ Nicholas Fantuzzi $^{1}$ [ $\cdot$ Francesco Fabbrocino $^{3} \cdot$ Raimondo Luciano $^{2}$
}

Received: 27 January 2021 / Accepted: 13 April 2021 / Published online: 27 April 2021

(c) The Author(s) 2021

\begin{abstract}
In this work, the bending behavior of nanoplates subjected to both sinusoidal and uniform loads in hygrothermal environment is investigated. The present plate theory is based on the classical laminated thin plate theory with strain gradient effect to take into account the nonlocality present in the nanostructures. The equilibrium equations have been carried out by using the principle of virtual works and a system of partial differential equations of the sixth order has been carried out, in contrast to the classical thin plate theory system of the fourth order. The solution has been obtained using a trigonometric expansion (e.g., Navier method) which is applicable to simply supported boundary conditions and limited lamination schemes. The solution is exact for sinusoidal loads; nevertheless, convergence has to be proved for other load types such as the uniform one. Both the effect of the hygrothermal loads and lamination schemes (cross-ply and angle-ply nanoplates) on the bending behavior of thin nanoplates are studied. Results are reported in dimensionless form and validity of the present methodology has been proven, when possible, by comparing the results to the ones from the literature (available only for cross-ply laminates). Novel applications are shown both for cross- and angle-ply laminated which can be considered for further developments in the same topic.
\end{abstract}

Keywords Kirchhoff plate theory $\cdot$ Nonlocal theory $\cdot$ Strain gradient theory $\cdot$ Hygrothermal load $\cdot$ Composite nanoplates · Cross- and angle-ply laminates

\section{Introduction}

Nanomaterials and nanostructures have been investigating recently for their innovative properties and features. The analysis and design of such materials and structures has increased rapidly in the last decades. Their application is extremely wide, and they can be used for different purposes [1-4]. Focusing on nanostructures several applications has been already presented in medicine [5], electronics [6], aerospace [7] and even in civil engineering [8-10]. The most common of such are nanoplates, nanoroads and nanobeams.

Technical Editor: João Marciano Laredo dos Reis.

Nicholas Fantuzzi

nicholas.fantuzzi@unibo.it

DICAM Department University of Bologna, Bologna, Italy

2 Engineering Department, Parthenope University, Naples, Italy

3 Department of Engineering, Telematic University Pegaso, Naples, Italy
However, classical continuum mechanics [11,12] is not sufficient to capture some effects which are present at the nanoscale and come from the influence of the microstructure on the macroscale, for instance, material constituent interactions create observable effects at the macroscale. As a matter of fact, it has been recently demonstrated that the behavior of nanostructures is affected by the material microstructure in [13-15], in addition, such effects have been also measured in experimental testing in $[16,17]$. Alternatively to experimental testing, numerical modeling can be employed such as atomic models [18-20]. However, such modeling solutions are computationally expensive with respect to continuum mechanics. Therefore, the main aim of the present study is to consider a higher-order continuum mechanics model which is able to take into consideration a length scale effects. This is the most common approach considered in the so-called nonlocal theories [21-24], where the description of the structural object is dependent on one of more length scale parameters.

Several nonlocal theories have been presented in the scientific community through the years such as couple stress 
[25], modified couple stress [26, 27], integral type and micropolar [28-30], strain and stress gradient [31-35] and modified strain gradient [36, 37],

This work focuses on a second-order strain gradient theory which is a simple and effective approach to investigate nonlocal effects in nanostructures. In the present case, the nonlocal effect is embodied in a single length scale parameter $\ell$ which multiplies the second gradient of the strain field; this leads to a stress field that is not only linearly dependent on the strain and also on its second gradient. This approach has been efficiently applied to isotropic [38] as well as composite structures [39]. Recent works on the static and dynamic analysis of nanostructures have been proposed. Timoshenko beam theory was combined with stress gradient theory for the bending phenomena of nanobeams made of functionally graded (FG) materials. Analogously Euler-Bernoulli beam theory has been used in [40] for the free and forced vibrations of nanobeams on elastic Pasternak foundation. Nonhomogeneous nanobeams on elastic medium have been analyzed with strain gradient effects by Civalek and Akgöz [41, 42]. Brischetto and co-workers used nonlocal theories for the study of composite plates and shells subjected to thermal, hygrometric and piezoelectric stress [43-46]. Nanoplate problems subjected to hygrothermal loads have been proposed in [47-52], using different nonlocal theories. Most common research works solve such nonlocal problems with analytical or semi-analytical methods which, in general, limit the analysis to simply supported conditions (for the Navier method [53]) or two sides supported and two arbitrary (for the Levy method [54]).

The aim of this study is to provide a trigonometric analytical and semi-analytical solutions to the bending problem of composite thin nanoplates subjected to hygrothermal using nonlocal second-order strain gradient theory. Sinusoidal and uniform loads for cross- and angle-ply laminates are studied, and for every uniform distribution considered also the convergence analysis for both displacement and stress fields is performed. This paper is structured as follows: after the introductory section, the theoretical background for Kirchhoff thin plates in hygrothermal environment is developed, using second-order strain gradient theory. Then, in order to validate the calculation code, implemented in MATLAB, various comparisons with the literature are reported [55-58]. After the comparisons, the results obtained for different lamination schemes and different types of loads are provided. Finally, remarks and conclusions are reported at the end of this paper.

\section{Theoretical background}

Consider a laminated thin nanoplate, modeled with the Kirchhoff plate theory, subjected to hygrothermal stresses [59]. The plate is composed of $k$ orthotropic layers oriented at angles $\theta_{1} ; \theta_{2} ; \ldots ; \theta_{k}$. The thickness of the $k$-th oriented layer, along the $z$ axis, is defined as $h_{k}=z_{k+1}-z_{k}$.

Introduced the reference system as in Fig. 1, we can define the displacement field of a generic point of the solid by means of the triad of displacement components $U, V, W$, which are functions of the coordinates $(x, y, z)$.

$\left\{\begin{array}{c}U \\ V \\ W\end{array}\right\}=\mathbb{\mathbb { V }}_{3} \mathbf{u}+z\left[\begin{array}{ccc}0 & 0 & \frac{\partial}{\partial x} \\ 0 & 0 & \frac{\partial}{\partial y} \\ 0 & 0 & 0\end{array}\right] \mathbf{u}$

where $\mathbf{u}^{\top}=\left\{\begin{array}{lll}u & v & w\end{array}\right\}$ represent the components of the displacement vector of a generic point placed on the reference surface of the plate. $\nabla_{3}$ is the $3 \times 3$ identity matrix.

From this model, we can trace the relationships between displacement components and deformation components that make up the compatibility equations.

$\varepsilon=\left\{\begin{array}{l}\varepsilon_{x x} \\ \varepsilon_{y y} \\ \gamma_{x y}\end{array}\right\}=\left\{\begin{array}{l}\varepsilon_{x x}^{(0)} \\ \varepsilon_{y y}^{(0)} \\ \gamma_{x y}^{(0)}\end{array}\right\}+z\left\{\begin{array}{c}\varepsilon_{x x}^{(1)} \\ \varepsilon_{y y}^{(1)} \\ \gamma_{x y}^{(1)}\end{array}\right\}$
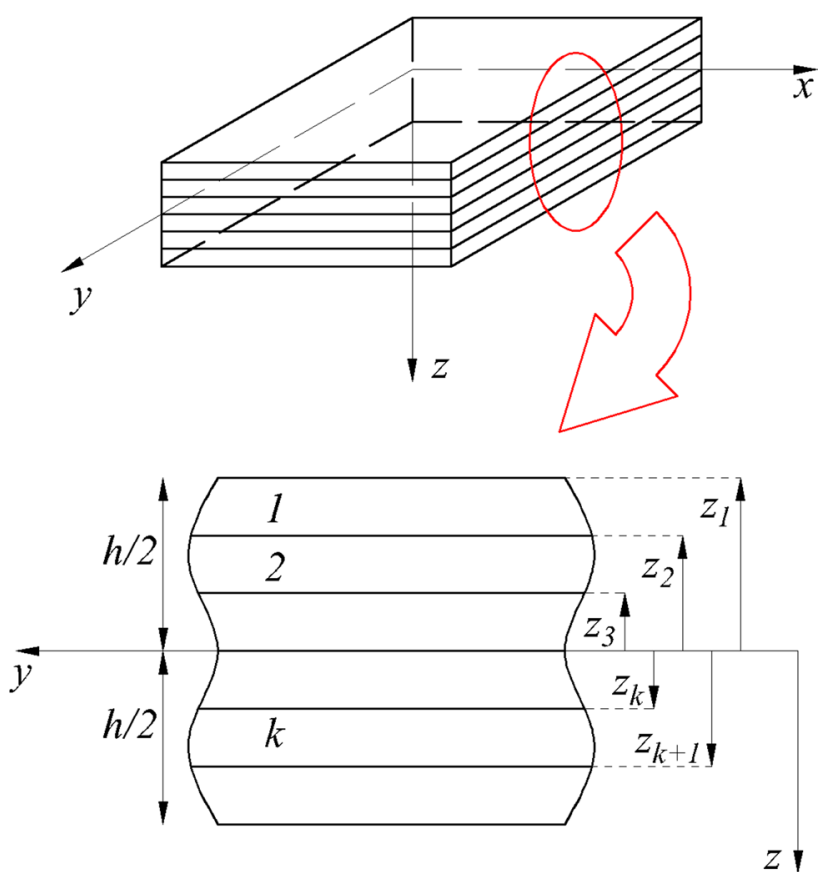

Fig. 1 Laminate general layout 
$\varepsilon=\varepsilon^{(0)}+z \varepsilon^{(1)}=\left(\mathbb{D}^{(0)}+z \mathbb{D}^{(1)}\right) \mathbf{u}$

where the superscript ${ }^{(0)}$ stands for membrane strain whereas (1) for bending strain, and

$$
\mathbb{D}^{(0)}=\left[\begin{array}{ccc}
\frac{\partial}{\partial x} & 0 & 0 \\
0 & \frac{\partial}{\partial y} & 0 \\
\frac{\partial}{\partial y} & \frac{\partial}{\partial x} & 0
\end{array}\right], \quad \mathbb{D}^{(1)}=\left[\begin{array}{ccc}
0 & 0 & -\frac{\partial^{2}}{\partial x^{2}} \\
0 & 0 & -\frac{\partial^{2}}{\partial y^{2}} \\
0 & 0 & -2 \frac{\partial^{2}}{\partial x \partial y}
\end{array}\right]
$$

We introduce the $Q_{i j}^{(k)}$ representing the rigidity of the orthotropic $k$-th ply into the plate reference system. The stiffnesses relate the stress components to the strain components, allowing to write the constitutive equations, and are defined through the following relationships as a function of engineering constants

$Q_{11}^{(k)}=\frac{E_{1}^{(k)}}{1-v_{12}^{(k)} v_{21}^{(k)}}, \quad Q_{22}^{(k)}=\frac{E_{2}^{(k)}}{1-v_{12}^{(k)} v_{21}^{(k)}}, \quad Q_{12}^{(k)}=\frac{v_{12}^{(k)} E_{2}^{(k)}}{1-v_{12}^{(k)} v_{21}^{(k)}}, \quad Q_{66}^{(k)}=G_{12}^{(k)}$

loads along the thickness is governed by the following relationships

$\Delta T=T_{0}+z T_{1} / h$

$\Delta C=C_{0}+z C_{1} / h$

where $T_{0}, C_{0}$ represent the constant distributions of temperature and humidity, analogously $T_{1}, C_{1}$ indicate the linear distributions of temperature and humidity. Note that all parameters are characterized by the same units since linear terms are multiplied by $z / h$.

By integrating the stresses along the thickness, we obtain:

In order to know the mechanical behavior of nanoplates subjected to hygrothermal stress, we introduce the nonlocal elastic theory of second-order strain gradient. Therefore, the constitutive equations take the following form

$$
\begin{aligned}
&\left\{\begin{array}{c}
\sigma_{x x}^{(k)} \\
\sigma_{y y}^{(k)} \\
\sigma_{x y}^{(k)}
\end{array}\right\}=\left(1-\ell^{2} \nabla^{2}\right)\left[\begin{array}{lll}
\bar{Q}_{11}^{(k)} & \bar{Q}_{12}^{(k)} & \bar{Q}_{16}^{(k)} \\
\bar{Q}_{12}^{(k)} & \bar{Q}_{22}^{(k)} & \bar{Q}_{26}^{(k)} \\
\bar{Q}_{16}^{(k)} & \bar{Q}_{26}^{(k)} & \bar{Q}_{66}^{(k)}
\end{array}\right]\left\{\begin{array}{c}
\varepsilon_{x x} \\
\varepsilon_{y y} \\
\gamma_{x y}
\end{array}\right\} \\
&-\left[\begin{array}{ccc}
\bar{Q}_{11}^{(k)} & \bar{Q}_{12}^{(k)} & \bar{Q}_{16}^{(k)} \\
\bar{Q}_{12}^{(k)} & \bar{Q}_{22}^{(k)} & \bar{Q}_{26}^{(k)} \\
\bar{Q}_{16}^{(k)} & \bar{Q}_{26}^{(k)} & \bar{Q}_{66}^{(k)}
\end{array}\right]\left\{\begin{array}{c}
\alpha_{x x}^{(k)} \Delta T+\beta_{x x}^{(k)} \Delta C \\
\alpha_{y y}^{(k)} \Delta T+\beta_{y y}^{(k)} \Delta C \\
2 \alpha_{x y}^{(k)} \Delta T+2 \beta_{x y}^{(k)} \Delta C
\end{array}\right\}
\end{aligned}
$$$$
\begin{aligned}
& \mathbf{N}=\left\{\begin{array}{l}
N_{x x} \\
N_{y y} \\
N_{x y}
\end{array}\right\}=\sum_{k=1}^{N_{L}} \int_{z_{k}}^{z_{k+1}}\left\{\begin{array}{c}
\sigma_{x x}^{(k)} \\
\sigma_{y y}^{(k)} \\
\sigma_{x y}^{(k)}
\end{array}\right\} \mathrm{d} z \\
& \mathbf{M}=\left\{\begin{array}{l}
M_{x x} \\
M_{y y} \\
M_{x y}
\end{array}\right\}=\sum_{k=1}^{N_{L}} \int_{z_{k}}^{z_{k+1}}\left\{\begin{array}{c}
\sigma_{x x}^{(k)} \\
\sigma_{y y}^{(k)} \\
\sigma_{x y}^{(k)}
\end{array}\right\} z \mathrm{~d} z
\end{aligned}
$$

Introducing the $\mathbf{A}, \mathbf{D}$ and $\mathbf{B}$ matrices, called membrane stiffness matrix, bending stiffness matrix and bending-membrane coupling stiffness matrix,

where $\bar{Q}_{i j}^{(k)}$ are the classical reduced elastic stiffnesses [55] in the geometric reference plane. The same can be done for the hygrothermal properties of the material as

$$
\begin{aligned}
\alpha_{x x}^{(k)} & =\alpha_{1}^{(k)} \cos ^{2} \theta^{(k)}+\alpha_{2}^{(k)} \sin ^{2} \theta^{(k)}, \quad \beta_{x x}^{(k)}=\beta_{1}^{(k)} \cos ^{2} \theta^{(k)}+\beta_{2}^{(k)} \sin ^{2} \theta^{(k)} \\
\alpha_{y y}^{(k)} & =\alpha_{1}^{(k)} \sin ^{2} \theta^{(k)}+\alpha_{2}^{(k)} \cos ^{2} \theta^{(k)}, \quad \beta_{y y}^{(k)}=\beta_{1}^{(k)} \sin ^{2} \theta^{(k)}+\beta_{2}^{(k)} \cos ^{2} \theta^{(k)} \\
2 \alpha_{x y}^{(k)} & =2\left(\alpha_{1}^{(k)}-\alpha_{2}^{(k)}\right) \sin \theta^{(k)} \cos \theta^{(k)}, \quad 2 \beta_{x y}^{(k)}=2\left(\beta_{1}^{(k)}-\beta_{2}^{(k)}\right) \sin \theta^{(k)} \cos \theta^{(k)}
\end{aligned}
$$

It is convenient to report the constitutive equation in matrix form as

$\boldsymbol{\sigma}^{(k)}=\left(1-\ell^{2} \nabla^{2}\right) \overline{\mathbf{Q}}^{(k)} \boldsymbol{\varepsilon}-\overline{\mathbf{Q}}^{(k)}\left(\boldsymbol{\alpha}^{(k)} T_{0}+\frac{z}{h} \boldsymbol{\alpha}^{(k)} T_{1}+\boldsymbol{\beta}^{(k)} C_{0}+\frac{z}{h} \boldsymbol{\beta}^{(k)} C_{1}\right)$

where $\ell$ is the nonlocal ratio and the operator $\nabla^{2}=\partial^{2} / \partial x^{2}+\partial^{2} / \partial y^{2}$. The variation of hygrothermal 


$$
\begin{aligned}
& \mathbf{A}=\sum_{k=1}^{N_{L}} \int_{z_{k}}^{z_{k+1}} \overline{\mathbf{Q}}^{(k)} \mathrm{d} z=\sum_{k=1}^{N_{L}} \overline{\mathbf{Q}}^{(k)}\left(z_{k+1}-z_{k}\right) \\
& \mathbf{B}=\sum_{k=1}^{N_{L}} \int_{z_{k}}^{z_{k+1}} \overline{\mathbf{Q}}^{(k)} z \mathrm{~d} z=\frac{1}{2} \sum_{k=1}^{N_{L}} \overline{\mathbf{Q}}^{(k)}\left(z_{k+1}^{2}-z_{k}^{2}\right) \\
& \mathbf{D}=\sum_{k=1}^{N_{L}} \int_{z_{k}}^{z_{k+1}} \overline{\mathbf{Q}}^{(k)} z^{2} \mathrm{~d} z=\frac{1}{3} \sum_{k=1}^{N_{L}} \overline{\mathbf{Q}}^{(k)}\left(z_{k+1}^{3}-z_{k}^{3}\right)
\end{aligned}
$$

and vectors $\mathbf{A}^{\alpha}, \mathbf{A}^{\beta}, \mathbf{B}^{\alpha}, \mathbf{B}^{\beta}, \mathbf{D}^{\alpha}$ and $\mathbf{D}^{\beta}$ containing the hygrothermal properties of the material

$$
\begin{aligned}
& \mathbf{A}^{\alpha}=\sum_{k=1}^{N_{L}} \int_{z_{k}}^{z_{k+1}} \overline{\mathbf{Q}}^{(k)} \boldsymbol{\alpha}^{(k)} \mathrm{d} z=\sum_{k=1}^{N_{L}} \overline{\mathbf{Q}}^{(k)} \boldsymbol{\alpha}^{(k)}\left(z_{k+1}-z_{k}\right) \\
& \mathbf{B}^{\alpha}=\sum_{k=1}^{N_{L}} \int_{z_{k}}^{z_{k+1}} \overline{\mathbf{Q}}^{(k)} \boldsymbol{\alpha}^{(k)} z \mathrm{~d} z=\frac{1}{2} \sum_{k=1}^{N_{L}} \overline{\mathbf{Q}}^{(k)} \boldsymbol{\alpha}^{(k)}\left(z_{k+1}^{2}-z_{k}^{2}\right) \\
& \mathbf{D}^{\alpha}=\sum_{k=1}^{N_{L}} \int_{z_{k}}^{z_{k+1}} \overline{\mathbf{Q}}^{(k)} \boldsymbol{\alpha}^{(k)} z^{2} \mathrm{~d} z=\frac{1}{3} \sum_{k=1}^{N_{L}} \overline{\mathbf{Q}}^{(k)} \boldsymbol{\alpha}^{(k)}\left(z_{k+1}^{3}-z_{k}^{3}\right) \\
& \mathbf{A}^{\beta}=\sum_{k=1}^{N_{L}} \int_{z_{k}}^{z_{k+1}} \overline{\mathbf{Q}}^{(k)} \boldsymbol{\beta}^{(k)} \mathrm{d} z=\sum_{k=1}^{N_{L}} \overline{\mathbf{Q}}^{(k)} \boldsymbol{\beta}^{(k)}\left(z_{k+1}-z_{k}\right) \\
& \mathbf{B}^{\beta}=\sum_{k=1}^{N_{L}} \int_{z_{k}}^{z_{k+1}} \overline{\mathbf{Q}}^{(k)} \boldsymbol{\beta}^{(k)} z \mathrm{~d} z=\frac{1}{2} \sum_{k=1}^{N_{L}} \overline{\mathbf{Q}}^{(k)} \boldsymbol{\beta}^{(k)}\left(z_{k+1}^{2}-z_{k}^{2}\right) \\
& \mathbf{D}^{\beta}=\sum_{k=1}^{N_{L}} \int_{z_{k}}^{z_{k+1}} \overline{\mathbf{Q}}^{(k)} \boldsymbol{\beta}^{(k)} z^{2} \mathrm{~d} z=\frac{1}{3} \sum_{k=1}^{N_{L}} \overline{\mathbf{Q}}^{(k)} \boldsymbol{\beta}^{(k)}\left(z_{k+1}^{3}-z_{k}^{3}\right)
\end{aligned}
$$

The stress characteristics as a function of the displacements take the following form

$$
\begin{aligned}
\mathbf{N}= & \left(\mathbf{A} \mathbb{D}^{(0)}+\mathbf{B} \mathbb{D}^{(1)}\right) \mathbf{u}-\ell^{2}\left[\mathbf{A}\left(\mathbb{D}_{x x}^{(0)}+\mathbb{D}_{y y}^{(0)}\right) \mathbf{u}+\mathbf{B}\left(\mathbb{D}_{x x}^{(1)}+\mathbb{D}_{y y}^{(1)}\right) \mathbf{u}\right]+ \\
& -\mathbf{A}^{\alpha} T_{0}-\frac{1}{h} \mathbf{B}^{\alpha} T_{1}-\mathbf{A}^{\beta} C_{0}-\frac{1}{h} \mathbf{B}^{\beta} C_{1} \\
\mathbf{M}= & \left(\mathbf{B} \mathbb{D}^{(0)}+\mathbf{D} \mathbb{D}^{(1)}\right) \mathbf{u}-\ell^{2}\left[\mathbf{B}\left(\mathbb{D}_{x x}^{(0)}+\mathbb{D}_{y y}^{(0)}\right) \mathbf{u}+\mathbf{D}\left(\mathbb{D}_{x x}^{(1)}+\mathbb{D}_{y y}^{(1)}\right) \mathbf{u}\right]+ \\
& -\mathbf{B}^{\alpha} T_{0}-\frac{1}{h} \mathbf{D}^{\alpha} T_{1}-\mathbf{B}^{\beta} C_{0}-\frac{1}{h} \mathbf{D}^{\beta} C_{1}
\end{aligned}
$$

where subscripts ${ }_{x x},{ }_{y y}$ represent second-order derivatives with respect to $x$ and $y$ applied to the operators defined in Eq. (4).

To obtain the balance equations we use the principle of virtual works $\delta U+\delta V=0$, where $\delta U$ is the variation of elastic energy and $\delta V$ is the potential of external work done by applied forces.

$$
\begin{aligned}
\delta U & =\sum_{k=1}^{N_{L}} \int_{\mathcal{A}} \int_{z_{k}}^{z_{k+1}} \delta \boldsymbol{\varepsilon}^{\top} \boldsymbol{\sigma}^{(k)} \mathrm{d} z \mathrm{~d} \mathcal{A} \\
& =\sum_{k=1}^{N_{L}} \int_{\mathcal{A}} \int_{z_{k}}^{z_{k+1}}\left(\mathbb{D}^{(0)} \delta \mathbf{u}\right)^{\top} \boldsymbol{\sigma}^{(k)}+\left(\mathbb{D}^{(1)} \delta \mathbf{u}\right)^{\top} z \boldsymbol{\sigma}^{(k)} \mathrm{d} z \mathrm{~d} \mathcal{A} \\
& =\int_{\mathcal{A}}\left(\mathbb{D}^{(0)} \delta \mathbf{u}\right)^{\top} \mathbf{N}+\left(\mathbb{D}^{(1)} \delta \mathbf{u}\right)^{\top} \mathbf{M} \mathrm{d} \mathcal{A}
\end{aligned}
$$

Integration by parts of the strain energy is

$$
\begin{aligned}
\delta U= & \int_{\mathcal{A}}\left(\mathbb{D}^{(0)} \delta \mathbf{u}\right)^{\top} \mathbf{N}+\left(\mathbb{D}^{(1)} \delta \mathbf{u}\right)^{\top} \mathbf{M} \mathrm{d} \mathcal{A} \\
= & \int_{\mathcal{A}}-\delta \mathbf{u}^{\top}\left(\mathbb{D}^{(0) \top} \mathbf{N}\right)-\delta \mathbf{u}^{\top}\left(\mathbb{D}^{(1) \top} \mathbf{M}\right) \mathrm{d} \mathcal{A} \\
& +\int_{\Gamma}\left[\delta \mathbf{u}^{\top}\left(\mathbf{n}^{(0) \top} \mathbf{N}\right)+\delta \mathbf{u}^{\top}\left(\left(\mathbb{D}^{(0)} \mathbf{n}^{(1)}\right)^{\top} \mathbf{M}\right)-\delta \mathbf{u}^{\top} \mathbb{D}^{(2) \top}\left(\mathbf{n}^{(0) \top} \mathbf{M}\right)\right] \mathrm{d} \mathcal{S}
\end{aligned}
$$

where

$$
\mathbf{n}^{(0)}=\left[\begin{array}{ccc}
n_{x} & 0 & 0 \\
0 & n_{y} & 0 \\
n_{y} & n_{x} & 0
\end{array}\right], \quad \mathbf{n}^{(1)}=\left[\begin{array}{ccc}
0 & 0 & n_{x} \\
0 & 0 & n_{y} \\
0 & 0 & 0
\end{array}\right], \quad \mathbb{D}^{(2)}=\left[\begin{array}{ccc}
0 & 0 & \frac{\partial}{\partial x} \\
0 & 0 & \frac{\partial}{\partial y} \\
0 & 0 & 0
\end{array}\right]
$$

Only transverse loads are applied to the plate, thus, potential of external work done by applied forces is 
$\delta V=-\int_{\mathcal{A}} \mathbf{q}^{\top} \delta \mathbf{u} \mathrm{d} \mathcal{A}$

where $\mathbf{q}=\left\{\begin{array}{lll}0 & 0 & q\end{array}\right\}^{\top}$.

The balance equations and the boundary condition result to be

$\mathbb{D}^{(0) \top} \mathbf{N}-\mathbb{D}^{(1) \top} \mathbf{M}-\mathbf{q}=\mathbf{0}$

$\mathbf{u}=0, \quad\left(\mathbf{n}^{(0) \top} \mathbf{N}\right)-\mathbb{D}^{(2) \top}\left(\mathbf{n}^{(0) \top} \mathbf{M}\right)=0$

$\mathbb{D}^{(2)} \mathbf{u}=0 \quad, \quad \mathbf{M}=0$

Replacing Eqs. (14) and (15) in Eq. (20), the strong form of the problem is obtained.

\section{Navier solution}

In this section, we introduce the Navier displacements field for an orthotropic cross-ply and angle-ply laminate. The solution is obtained by substituting the Navier displacements field in the balance equation.

$\left[\begin{array}{lll}\hat{c}_{11} & \hat{c}_{12} & \hat{c}_{13} \\ \hat{c}_{12} & \hat{c}_{22} & \hat{c}_{23} \\ \hat{c}_{13} & \hat{c}_{23} & \hat{c}_{33}\end{array}\right]\left\{\begin{array}{c}U_{m n} \\ V_{m n} \\ W_{m n}\end{array}\right\}=\left\{\begin{array}{c}0 \\ 0 \\ Q_{m n}\end{array}\right\}+\left\{\begin{array}{l}\mathcal{F}_{1, m n}^{\mathrm{T}} \\ \mathcal{F}_{2, m n}^{\mathrm{T}} \\ \mathcal{F}_{3, m n}^{\mathrm{T}}\end{array}\right\}$

The coefficients $\hat{c}_{i j}$ and $\mathcal{F}_{i, m n}^{\mathrm{T}}$ will be specified in the corresponding paragraphs for the specific case. Equation (22) can be solved by the method of static condensation. Consequently, the static solution is

$$
\begin{aligned}
W_{m n} & =\frac{1}{a_{m n}}\left[Q_{m n}+\mathcal{F}_{3, m n}^{\mathrm{T}}+\frac{a_{1}}{a_{0}} \mathcal{F}_{1, m n}^{\mathrm{T}}+\frac{a_{2}}{a_{0}} \mathcal{F}_{2, m n}^{\mathrm{T}}\right] \\
U_{m n} & =\frac{1}{a_{0}}\left[a_{1} W_{m n}+\mathcal{F}_{1, m n}^{\mathrm{T}} \hat{c}_{22}-\mathcal{F}_{2, m n}^{\mathrm{T}} \hat{c}_{12}\right] \\
V_{m n} & =\frac{1}{a_{0}}\left[a_{2} W_{m n}-\mathcal{F}_{1, m n}^{\mathrm{T}} \hat{c}_{12}+\mathcal{F}_{2, m n}^{\mathrm{T}} \hat{c}_{11}\right]
\end{aligned}
$$

where

$$
\begin{aligned}
a_{m n} & =\hat{c}_{33}+\hat{c}_{13} \frac{a_{1}}{a_{0}}+\hat{c}_{23} \frac{a_{2}}{a_{0}} \\
a_{0} & =\hat{c}_{11} \hat{c}_{22}+\hat{c}_{12} \hat{c}_{12} \\
a_{1} & =\hat{c}_{12} \hat{c}_{23}+\hat{c}_{13} \hat{c}_{22} \\
a_{2} & =\hat{c}_{13} \hat{c}_{12}+\hat{c}_{11} \hat{c}_{23} .
\end{aligned}
$$

\subsection{Cross-ply laminate}

In this section, the analytical solution for cross-ply laminates is developed. The simply supported boundary condition for cross-ply laminates result to be:

$$
\begin{aligned}
u(x, 0)=u(x, b) & =0 \quad ; \quad v(0, y)=v(a, y)=0 \\
w(x, 0)=w(x, b) & =0 \quad ; \quad w(0, y)=w(a, y)=0 \\
\left.\frac{\partial w}{\partial x}\right|_{(x, 0)} & =\left.\frac{\partial w}{\partial x}\right|_{(x, b)}=0 \quad ;\left.\quad \frac{\partial w}{\partial y}\right|_{(0, y)}=\left.\frac{\partial w}{\partial y}\right|_{(a, y)}=0
\end{aligned}
$$

In order to satisfy the boundary condition, Navier displacements field is assumed to be

$$
\begin{gathered}
u(x, y)=\sum_{m=1}^{\infty} \sum_{n=1}^{\infty} U_{m n} \cos \alpha x \sin \beta y \\
v(x, y)=\sum_{m=1}^{\infty} \sum_{n=1}^{\infty} V_{m n} \sin \alpha x \cos \beta y \\
w(x, y)=\sum_{m=1}^{\infty} \sum_{n=1}^{\infty} W_{m n} \sin \alpha x \sin \beta y
\end{gathered}
$$

A trigonometric development is also used for the mechanical and hygrothermal loads shown as

$q(x, y)=\sum_{m=1}^{\infty} \sum_{n=1}^{\infty} Q_{m n} \sin \alpha x \sin \beta y$

$\left(T_{0}(x, y), T_{1}(x, y)\right)=\sum_{m=1}^{\infty} \sum_{n=1}^{\infty}\left(T_{0, m n}, T_{1, m n}\right) \sin \alpha x \sin \beta y$

$\left(C_{0}(x, y), C_{1}(x, y)\right)=\sum_{m=1}^{\infty} \sum_{n=1}^{\infty}\left(C_{0, m n}, C_{1, m n}\right) \sin \alpha x \sin \beta y$

where $\alpha=m \pi / a$ e $\beta=n \pi / b$. The coefficients $\hat{c}_{i j}$ for the cross-ply laminate are [39]

$$
\begin{aligned}
\hat{c}_{11}= & \alpha^{2} A_{11}+\beta^{2} A_{66}+\ell^{2}\left[\alpha^{4} A_{11}+\alpha^{2} \beta^{2}\left(A_{11}+A_{66}\right)+\beta^{4} A_{66}\right] \\
\hat{c}_{12}= & \alpha \beta\left(A_{12}+A_{66}\right)+\ell^{2}\left[\alpha^{3} \beta\left(A_{12}+A_{66}\right)+\alpha \beta^{3}\left(A_{12}+A_{66}\right)\right] \\
\hat{c}_{13}= & -\alpha^{3} B_{11}-\alpha \beta^{2}\left(B_{12}+2 B_{66}\right) \\
& -\ell^{2}\left[\alpha^{5} B_{11}+\alpha^{3} \beta^{2}\left(B_{12}+B_{11}+2 B_{66}\right)+\alpha \beta^{4}\left(B_{12}+2 B_{66}\right)\right] \\
\hat{c}_{22}= & \beta^{2} A_{22}+\alpha^{2} A_{66}+\ell^{2}\left[\alpha^{2} \beta^{2}\left(A_{22}+A_{66}\right)+\alpha^{4} A_{66}+\beta^{4} A_{22}\right] \\
\hat{c}_{23}= & -\alpha^{2} \beta\left(B_{12}+2 B_{66}\right)-\beta^{3} B_{22} \\
& -\ell^{2}\left[\alpha^{4} \beta\left(B_{12}+2 B_{66}\right)+\alpha^{2} \beta^{3}\left(B_{22}+B_{12}+2 B_{66}\right)+\beta^{5} B_{22}\right] \\
\hat{c}_{33}= & \alpha^{4} D_{11}+2 \alpha^{2} \beta^{2}\left(D_{12}+2 D_{66}\right)+\beta^{4} D_{22} \\
& +\ell^{2}\left[\alpha^{6} D_{11}+\alpha^{4} \beta^{2}\left(D_{11}+2 D_{12}+4 D_{66}\right)\right. \\
& \left.+\alpha^{2} \beta^{4}\left(D_{22}+2 D_{12}+4 D_{66}\right)+\beta^{6} D_{22}\right]
\end{aligned}
$$

The hygrothermal load vector have the following form 
Table 1 Displacements $(\bar{w})$ of a cross-ply square plate simply supported, subjected to a sinusoidal temperature distribution

Table 2 Displacements $(\bar{w})$ and stresses $(\bar{\sigma})$ of a cross-ply $(0 / 90)$ square plate, simply supported, subjected to a sinusoidal temperature distribution

\begin{tabular}{lll}
\hline & Reddy [55] & Present \\
\hline$(0)$ & 1.0312 & 1.0312 \\
$(0 / 90)$ & 1.1504 & 1.1504 \\
$(0 / 90 / 0)$ & 1.0312 & 1.0312 \\
$(0 / 90)_{5}$ & 1.0331 & 1.0331 \\
\hline
\end{tabular}

\begin{tabular}{lll}
\hline & Sayyad [58] & Present \\
\hline $\bar{w}$ & 1.1504 & 1.1504 \\
$\bar{\sigma}_{x x}$ & 0.307 & 0.3074 \\
$\bar{\sigma}_{y y}$ & 0.307 & 0.3074 \\
\hline
\end{tabular}

Table 3 Displacements $(\bar{w})$ of a cross-play square plate, simply supported, subjected to a sinusoidal temperature distribution, assuming that the thermal properties of the material do not depend on the effective disposition of the laminae (Zenkour, FPT, HPT and SPT) are the theories considered in [56])

\begin{tabular}{llllll}
\hline & \multicolumn{2}{l}{ Zenkour [56] } & & \\
\cline { 2 - 5 } & Zenkour & FPT & HPT & SPT & Present \\
\hline$(0)$ & 1.0313 & 1.0313 & 1.0313 & 1.0313 & 1.0312 \\
$(0 / 90)$ & 1.6765 & 1.6765 & 1.6766 & 1.6766 & 1.6765 \\
$(0 / 90 / 0)$ & 1.0949 & 1.0949 & 1.0950 & 1.0950 & 1.0944 \\
\hline
\end{tabular}

$$
\begin{aligned}
\mathcal{F}_{1, m n}^{\mathrm{T}} & =-\alpha\left[\left(A_{1}^{\alpha} T_{0, m n}+\frac{1}{h} B_{1}^{\alpha} T_{1, m n}\right)+\left(A_{1}^{\beta} C_{0, m n}+\frac{1}{h} B_{1}^{\beta} C_{1, m n}\right)\right] \\
\mathcal{F}_{2, m n}^{\mathrm{T}} & =-\beta\left[\left(A_{2}^{\alpha} T_{0, m n}+\frac{1}{h} B_{2}^{\alpha} T_{1, m n}\right)+\left(A_{2}^{\beta} C_{0, m n}+\frac{1}{h} B_{2}^{\beta} C_{1, m n}\right)\right] \\
\mathcal{F}_{3, m n}^{\mathrm{T}} & =\alpha^{2}\left[\left(B_{1}^{\alpha} T_{0, m n}+\frac{1}{h} D_{1}^{\alpha} T_{1, m n}\right)+\left(B_{1}^{\beta} C_{0, m n}+\frac{1}{h} D_{1}^{\beta} C_{1, m n}\right)\right] \\
& +\beta^{2}\left[\left(B_{2}^{\alpha} T_{0, m n}+\frac{1}{h} D_{2}^{\alpha} T_{1, m n}\right)+\left(B_{2}^{\beta} C_{0, m n}+\frac{1}{h} D_{2}^{\beta} C_{1, m n}\right)\right]
\end{aligned}
$$

Table 5 Displacements $(\bar{w})$ and stresses $(\bar{\sigma})$ of a simply supported square cross-ply nanoplates, with different layout and for different values of nonlocal parameter, subjected to a sinusoidal temperature distribution $\left(T_{0}=0\right)$

\begin{tabular}{lllrrl}
\hline$(\ell / a)^{2}$ & & $\bar{w}$ & \multicolumn{1}{l}{$\bar{\sigma}_{x x}$} & \multicolumn{1}{l}{$\bar{\sigma}_{y y}$} & \multicolumn{1}{l}{$\bar{\sigma}_{x y}$} \\
\hline 0 & $(0)$ & 1.0312 & 0.0263 & -0.9914 & 0.5089 \\
& $(0 / 90)$ & 1.1504 & -0.3074 & 0.3074 & 0.5677 \\
& $(0 / 90 / 0)$ & 1.0312 & 0.0263 & -0.9914 & 0.5089 \\
& $(0 / 90)_{2}$ & 1.0454 & 0.1241 & -0.1241 & 0.5159 \\
0.05 & $(0)$ & 0.5190 & 6.4245 & -1.3081 & 0.2561 \\
& $(0 / 90)$ & 0.5790 & 6.2566 & -6.2566 & 0.2857 \\
& $(0 / 90 / 0)$ & 0.5190 & 6.4245 & -1.3081 & 0.2561 \\
& $(0 / 90)_{2}$ & 0.5261 & 6.4737 & -6.4737 & 0.2596 \\
0.10 & $(0)$ & 0.3467 & 8.5760 & -1.4147 & 0.1711 \\
& $(0 / 90)$ & 0.3868 & 8.4638 & -8.4638 & 0.1909 \\
& $(0 / 90 / 0)$ & 0.3467 & 8.5760 & -1.4147 & 0.1711 \\
& $(0 / 90)_{2}$ & 0.3515 & 8.6089 & -8.6089 & 0.1735 \\
\hline
\end{tabular}

The Navier solution for cross-ply laminate with simply supported boundary condition is valid only if: $A_{16}=A_{26}=B_{16}=B_{26}=D_{16}=D_{26}=0$; thus, it can be developed for laminates with a single generally orthotropic layer, symmetrically laminated plates with multiple specially orthotropic layers and antisymmetric cross-ply laminated plates.

\subsection{Angle-ply laminate}

In this section, the analytical solution for angle-ply laminates is developed. The simply supported boundary condition for angle-ply laminates results to be:

\begin{tabular}{|c|c|c|c|c|}
\hline \multirow[t]{3}{*}{$a / b$} & \multicolumn{3}{|c|}{ Zenkour [57] } & \\
\hline & \multicolumn{4}{|c|}{ Thermal load $\left(T_{0}=0, T_{1}=100 ; C_{0}=0, C_{1}=0\right)$} \\
\hline & FPT & HPT & SPT & Present \\
\hline 0.5 & 1.07408 & 1.07413 & 1.07414 & 1.07384 \\
\hline 1.0 & 1.09485 & 1.09497 & 1.09499 & 1.09438 \\
\hline 2.0 & 0.76433 & 0.76424 & 0.76423 & 0.76452 \\
\hline \multirow[t]{2}{*}{$a / b$} & \multicolumn{4}{|c|}{ Hygrothermal load $\left(T_{0}=0, T_{1}=100 ; C_{0}=0, C_{1}=3 \times 10^{-4}\right)$} \\
\hline & FPT & HPT & SPT & Present \\
\hline 0.5 & 1.11308 & 1.11316 & 1.11317 & 1.11271 \\
\hline 1.0 & 1.19565 & 1.19587 & 1.19590 & 1.19477 \\
\hline 2.0 & 0.92694 & 0.92696 & 0.92697 & 0.92684 \\
\hline
\end{tabular}

$$
\begin{aligned}
u(0, y)=u(a, y)=0 & ; \quad v(x, 0)=v(x, b)=0 \\
w(x, 0)=w(x, b)=0 & ; \quad w(0, y)=w(a, y)=0 \\
\left.\frac{\partial w}{\partial x}\right|_{(x, 0)}=\left.\frac{\partial w}{\partial x}\right|_{(x, b)}=0 & ;\left.\quad \frac{\partial w}{\partial y}\right|_{(0, y)}=\left.\frac{\partial w}{\partial y}\right|_{(a, y)}=0
\end{aligned}
$$

Table 4 Displacement $(\bar{w})$ of a laminate $(0 / 90 / 0)$ for different kind of load and for different values of ratio $a / b$ 
Fig. 2 Displacements $(\bar{w})$ of cross-ply nanoplates $(0 / 90)$ (a) and $(0 / 90)_{2}$ (b) subjected to sinusoidal thermal load, for different values of $a / b$ ratio and nonlocal parameter $(\ell / a)^{2}$

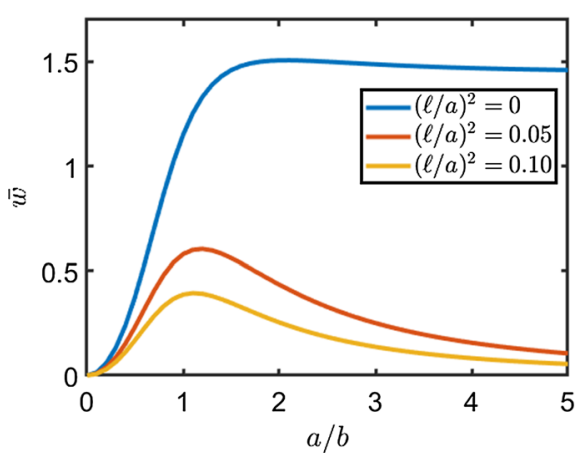

(a)

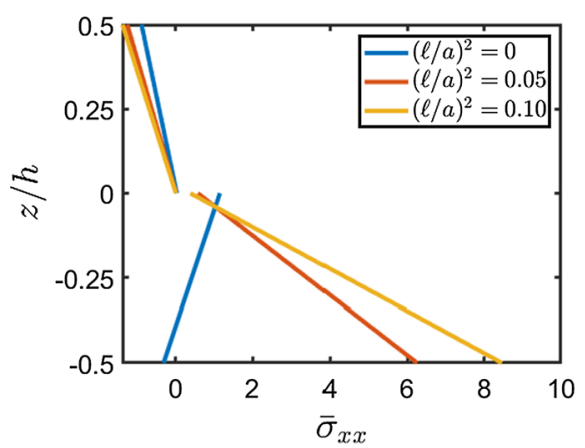

(a)

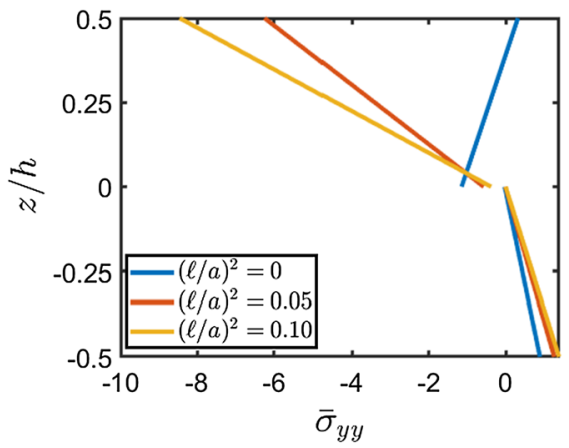

(b)

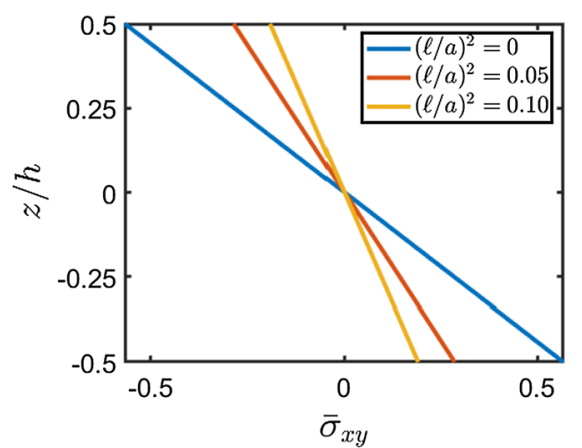

(c)

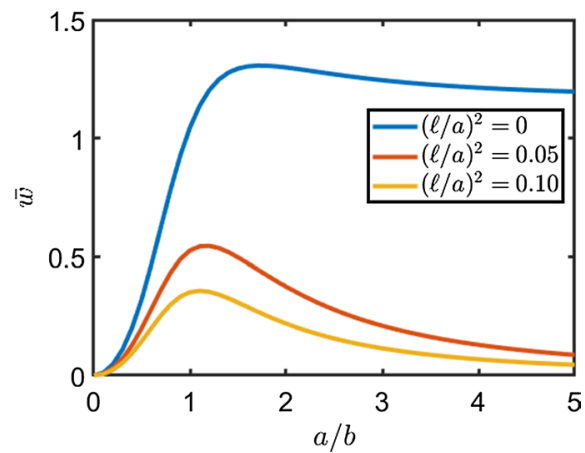

(b)

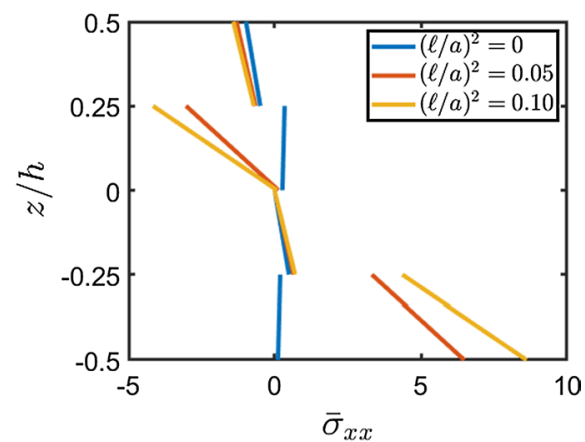

(d)

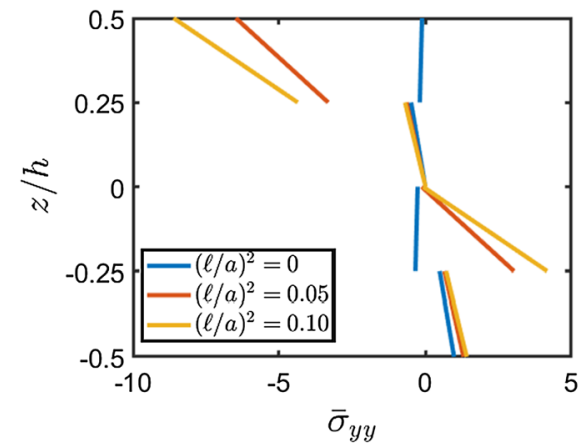

(e)

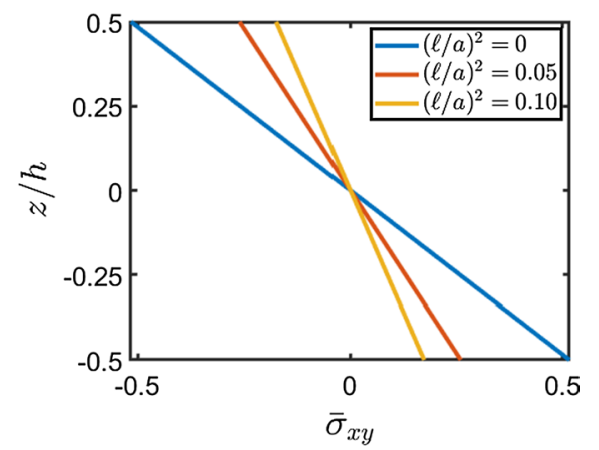

(f) 


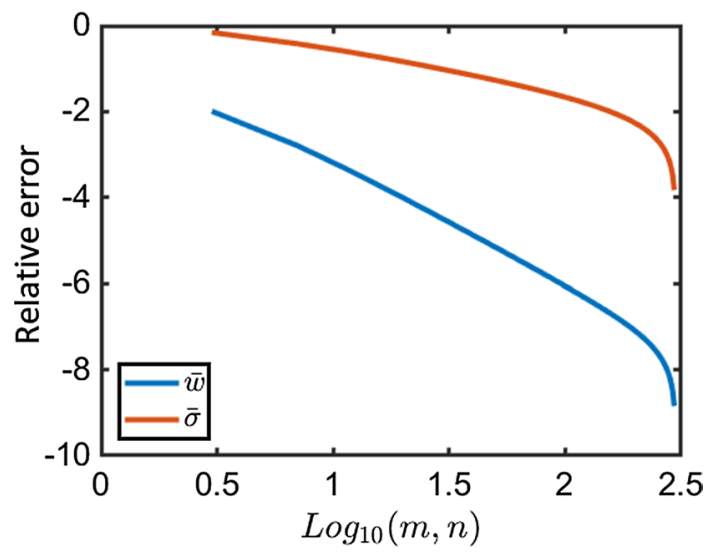

Fig. 4 Relative error in logarithmic scale by varying $n, m$ for the uniform thermal load

Table 6 Displacements $(\bar{w})$ and stresses $(\bar{\sigma})$ of a simply supported square cross-ply nanoplates, with different layout and for different values of nonlocal parameter, subjected to a uniform temperature load distribution $\left(m, n=1,3,5, \ldots, 199 ; T_{0}=0\right)$

\begin{tabular}{lllrrl}
\hline$(\ell / a)^{2}$ & & \multicolumn{1}{l}{$\bar{l}$} & \multicolumn{1}{l}{$\bar{\sigma}_{x x}$} & \multicolumn{1}{l}{$\bar{\sigma}_{y y}$} \\
\hline 0 & $(0)$ & 1.4334 & -1.8854 & -1.3228 & 4.3043 \\
& $(0 / 90)$ & 1.7296 & -1.9998 & 1.9998 & 3.5070 \\
& $(0 / 90 / 0)$ & 1.4862 & -2.5797 & -1.1460 & 3.7548 \\
& $(0 / 90)_{2}$ & 1.5823 & -2.2999 & 2.2999 & 3.0573 \\
0.05 & $(0)$ & 0.7966 & 3.6172 & -1.2545 & 0.5341 \\
& $(0 / 90)$ & 0.9126 & 3.4753 & -3.4753 & 0.5437 \\
& $(0 / 90 / 0)$ & 0.8065 & 3.4880 & -1.2151 & 0.5118 \\
& $(0 / 90)_{2}$ & 0.8313 & 3.6223 & -3.6223 & 0.4894 \\
0.10 & $(0)$ & 0.5376 & 6.5226 & -1.3572 & 0.3409 \\
& $(0 / 90)$ & 0.6128 & 6.4163 & -6.4163 & 0.3523 \\
& $(0 / 90 / 0)$ & 0.5430 & 6.4518 & -1.3355 & 0.3289 \\
& $(0 / 90)_{2}$ & 0.5580 & 6.5357 & -6.5357 & 0.3177 \\
\hline
\end{tabular}

In order to satisfy the boundary condition, the Navier displacements field is assumed to be

$$
\begin{aligned}
& u(x, y)=\sum_{m=1}^{\infty} \sum_{n=1}^{\infty} U_{m n} \sin \alpha x \cos \beta y \\
& v(x, y)=\sum_{m=1}^{\infty} \sum_{n=1}^{\infty} V_{m n} \cos \alpha x \sin \beta y \\
& w(x, y)=\sum_{m=1}^{\infty} \sum_{n=1}^{\infty} W_{m n} \sin \alpha x \sin \beta y
\end{aligned}
$$

It is similar to what was done before the loads are (27), (28), (29).

The coefficients $\hat{c}_{i j}$ for the angle-ply laminate are [39]

$$
\begin{aligned}
\hat{c}_{11}= & \alpha^{2} A_{11}+\beta^{2} A_{66}+\ell^{2}\left[\alpha^{4} A_{11}+\alpha^{2} \beta^{2}\left(A_{11}+A_{66}\right)+\beta^{4} A_{66}\right] \\
\hat{c}_{12}= & \alpha \beta\left(A_{12}+A_{66}\right)+\ell^{2}\left[\alpha^{3} \beta\left(A_{12}+A_{66}\right)+\alpha \beta^{3}\left(A_{12}+A_{66}\right)\right] \\
\hat{c}_{13}= & -3 \alpha^{2} \beta B_{16}-\beta^{3} B_{26}-\ell^{2}\left[3 B_{16}\left(\alpha^{4} \beta+\alpha^{2} \beta^{3}\right)+B_{26}\left(\alpha^{2} \beta^{3}+\beta^{5}\right)\right] \\
\hat{c}_{22}= & \beta^{2} A_{22}+\alpha^{2} A_{66}+\ell^{2}\left[\alpha^{2} \beta^{2}\left(A_{22}+A_{66}\right)+\alpha^{4} A_{66}+\beta^{4} A_{22}\right] \\
\hat{c}_{23}= & -\alpha^{3} B_{16}-3 \alpha \beta^{2} B_{26}-\ell^{2}\left[B_{16}\left(\alpha^{5}+\alpha^{3} \beta^{2}\right)+3 B_{26}\left(\alpha \beta^{4}+\alpha^{3} \beta^{2}\right)\right] \\
\hat{c}_{33}= & \alpha^{4} D_{11}+2 \alpha^{2} \beta^{2}\left(D_{12}+2 D_{66}\right)+\beta^{4} D_{22} \\
& +\ell^{2}\left[\alpha^{6} D_{11}+\alpha^{4} \beta^{2}\left(D_{11}+2 D_{12}+4 D_{66}\right)\right. \\
& \left.+\alpha^{2} \beta^{4}\left(D_{22}+2 D_{12}+4 D_{66}\right)+\beta^{6} D_{22}\right]
\end{aligned}
$$

The hygrothermal load vector have the following form:

$$
\begin{aligned}
\mathcal{F}_{1, m n}^{\mathrm{T}} & =-\beta\left[\left(A_{3}^{\alpha} T_{0, m n}+\frac{1}{h} B_{3}^{\alpha} T_{1, m n}\right)+\left(A_{3}^{\beta} C_{0, m n}+\frac{1}{h} B_{3}^{\beta} C_{1, m n}\right)\right] \\
\mathcal{F}_{2, m n}^{\mathrm{T}} & =-\alpha\left[\left(A_{3}^{\alpha} T_{0, m n}+\frac{1}{h} B_{3}^{\alpha} T_{1, m n}\right)+\left(A_{3}^{\beta} C_{0, m n}+\frac{1}{h} B_{3}^{\beta} C_{1, m n}\right)\right] \\
\mathcal{F}_{3, m n}^{\mathrm{T}} & =\alpha^{2}\left[\left(B_{1}^{\alpha} T_{0, m n}+\frac{1}{h} D_{1}^{\alpha} T_{1, m n}\right)+\left(B_{1}^{\beta} C_{0, m n}+\frac{1}{h} D_{1}^{\beta} C_{1, m n}\right)\right] \\
& +\beta^{2}\left[\left(B_{2}^{\alpha} T_{0, m n}+\frac{1}{h} D_{2}^{\alpha} T_{1, m n}\right)+\left(B_{2}^{\beta} C_{0, m n}+\frac{1}{h} D_{2}^{\beta} C_{1, m n}\right)\right]
\end{aligned}
$$

Fig. 5 Displacements $(\bar{w})$ of cross-ply nanoplates $(0 / 90)$ (a) and $(0 / 90)_{2}$ (b) subjected to uniform thermal load, for different values of $a / b$ ratio and nonlocal parameter $(\ell / a)^{2}$

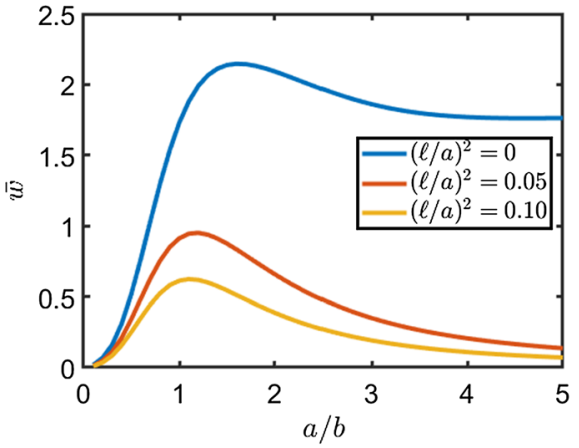

(a)

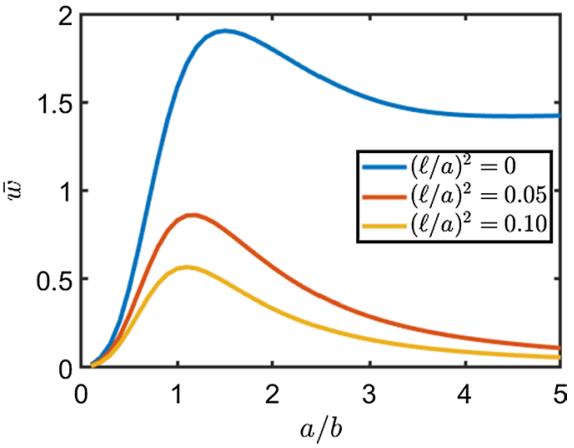

(b) 
Fig. 6 Stresses $(\bar{\sigma})$ of square plates $(0 / 90)(\mathbf{a}-\mathbf{c})$ and $(0 / 90)_{2}$ (d-f) subjected to uniformal thermal load, for different nonlocal parameters $(\ell / a)^{2}$

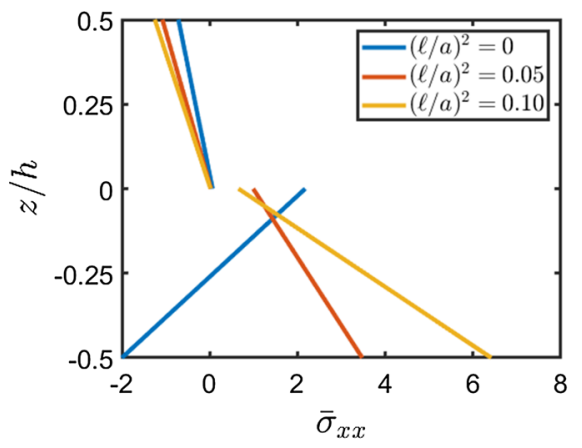

(a)

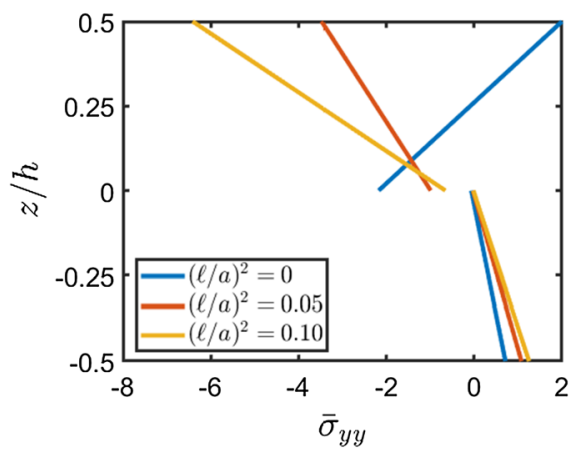

(b)

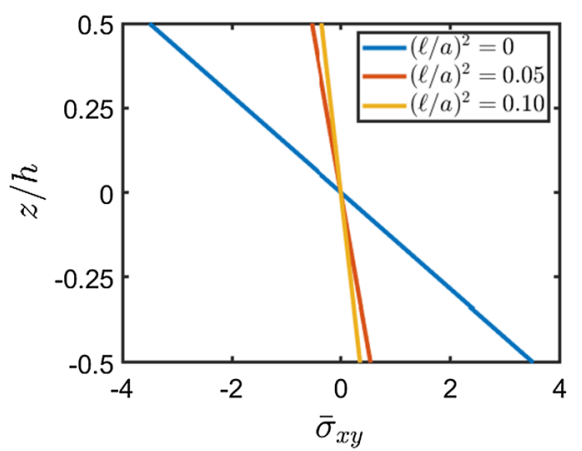

(c)

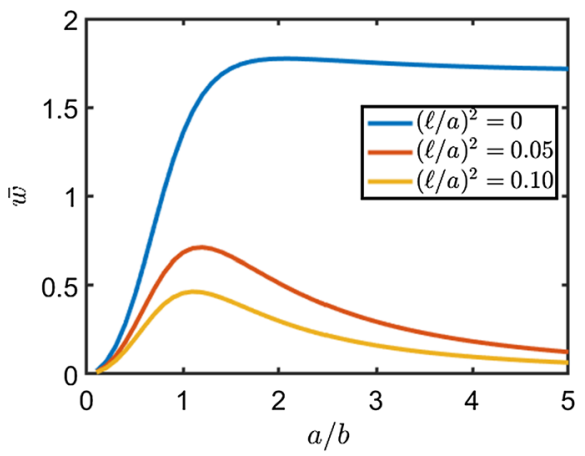

(a)

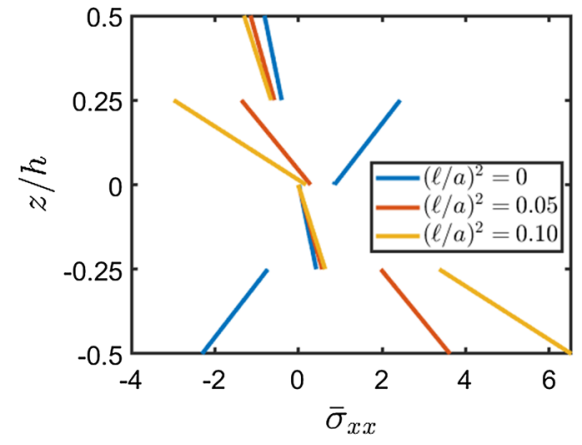

(d)

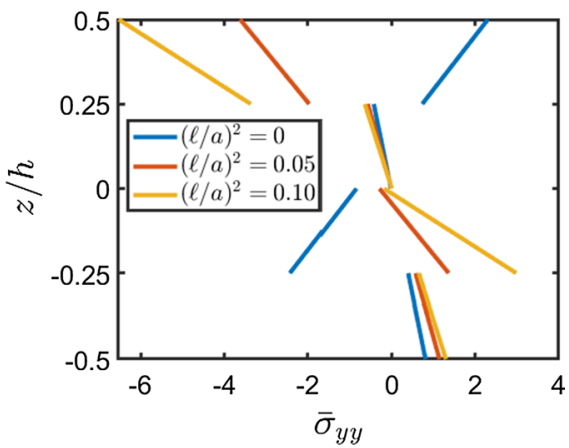

(e)

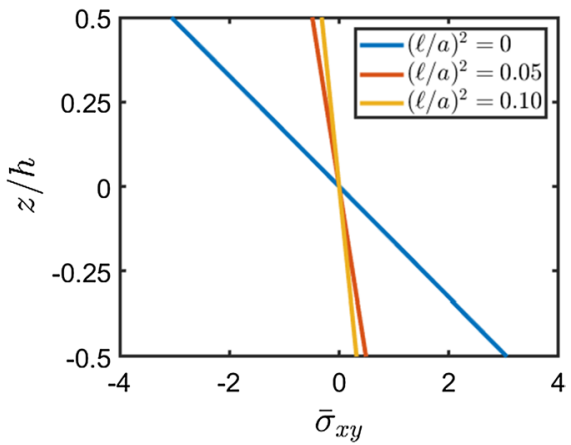

(f)

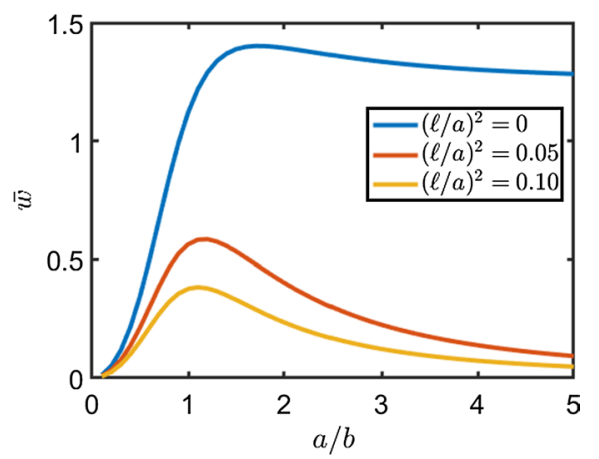

(b) 
Table 7 Displacements $(\bar{w})$ and stresses $(\bar{\sigma})$ of a simply supported square cross-ply nanoplates, with different layout and for different values of nonlocal parameter, subjected to a sinusoidal hygrothermal load distribution $\left(T_{0}=C_{0}=0\right)$

\begin{tabular}{lllrrl}
\hline$(\ell / a)^{2}$ & & \multicolumn{1}{l}{$\bar{l}$} & \multicolumn{1}{l}{$\bar{\sigma}_{x x}$} & \multicolumn{1}{l}{$\bar{\sigma}_{y y}$} \\
\hline 0 & $(0)$ & 1.0898 & -0.5411 & -1.6168 & 0.5378 \\
& $(0 / 90)$ & 1.3563 & -1.2873 & 1.2873 & 0.6693 \\
& $(0 / 90 / 0)$ & 1.0898 & -0.5411 & -1.6168 & 0.5378 \\
& $(0 / 90)_{2}$ & 1.1216 & -0.3225 & 0.3225 & 0.5535 \\
0.05 & $(0)$ & 0.5485 & 6.2211 & -1.9515 & 0.2707 \\
& $(0 / 90)$ & 0.6826 & 5.8456 & -5.8456 & 0.3369 \\
& $(0 / 90 / 0)$ & 0.5485 & 6.2211 & -1.9515 & 0.2707 \\
& $(0 / 90)_{2}$ & 0.5645 & 6.3311 & -6.3311 & 0.2786 \\
0.10 & $(0)$ & 0.3665 & 8.4949 & -2.0641 & 0.1808 \\
& $(0 / 90)$ & 0.4561 & 8.2440 & -8.2440 & 0.2251 \\
& $(0 / 90 / 0)$ & 0.3665 & 8.4949 & -2.0641 & 0.1808 \\
& $(0 / 90)_{2}$ & 0.3771 & 8.5685 & -8.5685 & 0.1861 \\
\hline
\end{tabular}

Fig. 8 Stresses $(\bar{\sigma})$ of square plates $(0 / 90)(\mathbf{a}-\mathbf{c})$ and $(0 / 90)_{2}$ (d-f) subjected to sinusoidal hygrothermal load, for different nonlocal parameters $(\ell / a)^{2}$
The Navier solution for angle ply laminate with simply supported boundary condition is valid only if: $A_{16}=A_{26}=B_{11}=B_{12}=B_{22}=B_{66}=D_{16}=D_{26}=0$; thus, it can be developed for laminates with a single generally orthotropic layer, symmetrically laminated plates with multiple specially orthotropic layers and antisymmetric angle-ply laminated plates.

\section{Results and discussion}

In this section, the analytical solutions for cross- and angleply laminates subjected to thermal and hygrothermal loads are carried out. For each study case, the comparison between classical and nonlocal theory is shown. The properties of the

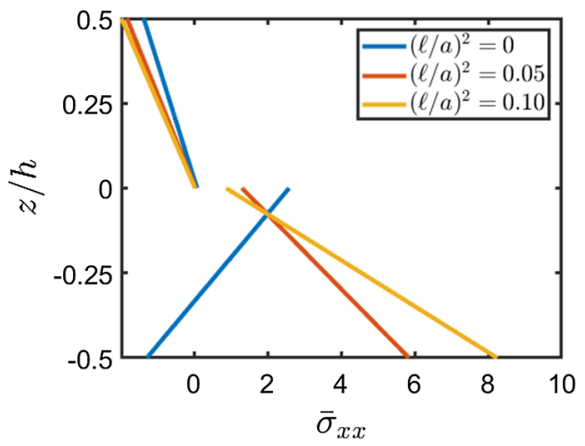

(a)

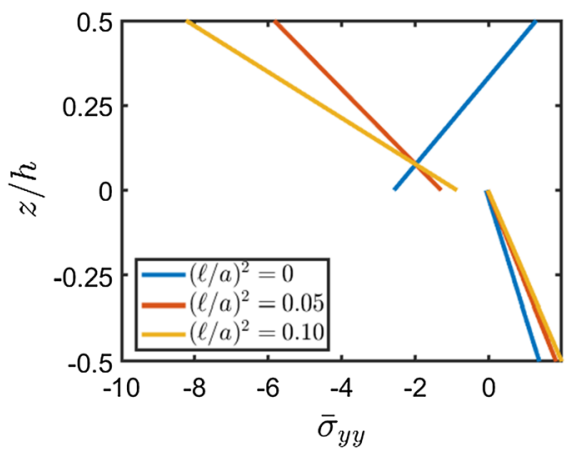

(b)

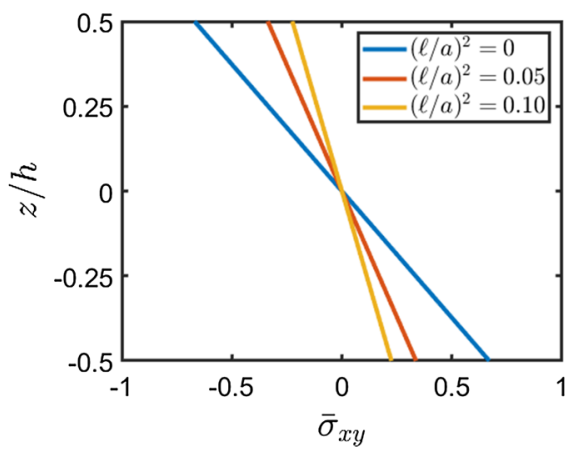

(c)

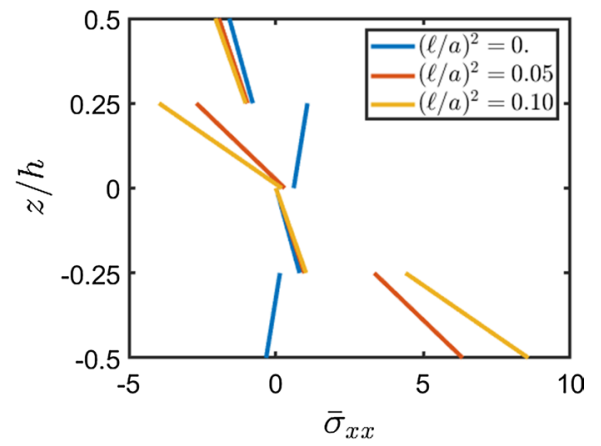

(d)

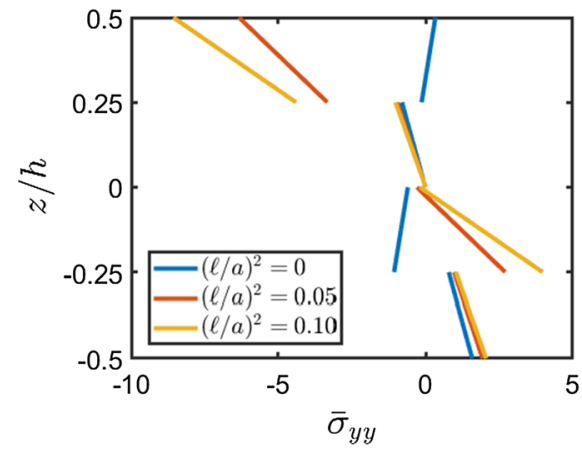

(e)

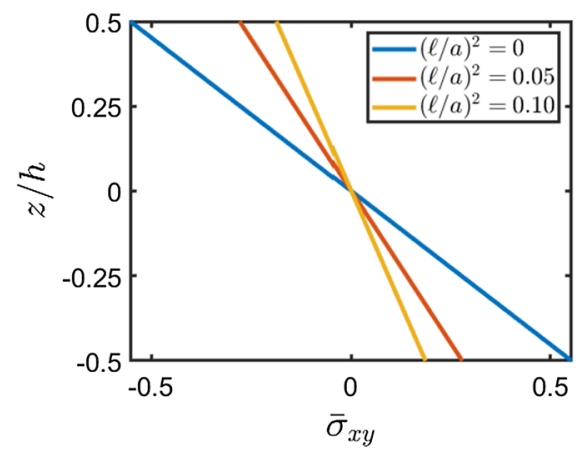

(f) 


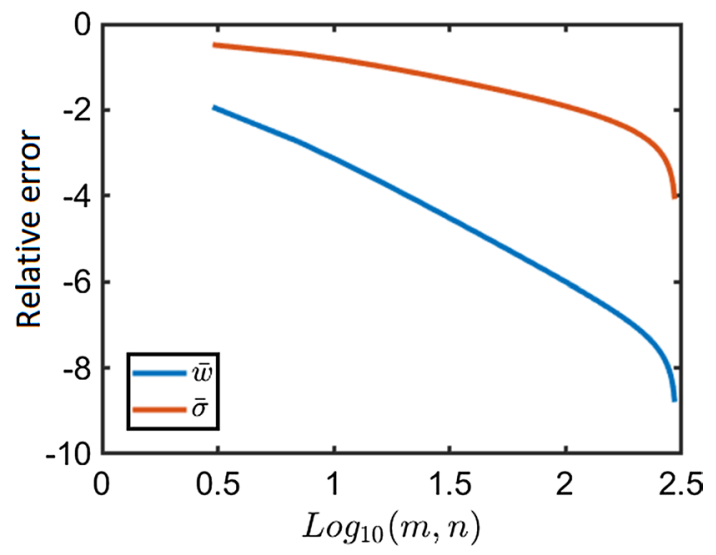

Fig. 9 Relative error in logarithmic by varying of $n, m$ for uniform hygrothermal load

material considered for the numerical solution are $E_{1}=25$, $E_{2}=1, G_{12}=0.5, v_{12}=0.25, \alpha_{2} / \alpha_{1}=3, \alpha_{1}=10^{-6}, \beta_{1}=0$, $\beta_{2}=0.44$. Please note that the units of measures are not reported because a consistent system of units has been used. The plates considered are rectangular with a ratio $a / h=100$ and the total height of the laminate is kept constant independently of the number of plies from which it is composed. Initially, comparisons were made with the results found in the scientific literature. The formulas used to normalize the results and the point at which they are calculated are shown as

$$
\begin{array}{ccc}
\bar{w}=w(a / 2, b / 2) \frac{10 h}{\alpha_{1} T_{1} b^{2}}, & \bar{\sigma}_{x y}=\frac{\sigma_{x y}(a, b,-h / 2)}{E_{2} \alpha_{1} T_{1} b} \\
\bar{\sigma}_{x x}=\frac{\sigma_{x x}(a / 2, b / 2,-h / 2)}{E_{2} \alpha_{1} T_{1} b}, & \bar{\sigma}_{y y}=\frac{\sigma_{y y}(a / 2, b / 2, h / 2)}{E_{2} \alpha_{1} T_{1} b}
\end{array}
$$

Table 8 Displacements $(\bar{w})$ and stresses $(\bar{\sigma})$ of a simply supported square cross-ply nanoplates, with different layout and for different values of nonlocal parameter, subjected to an uniform hygrothermal load distribution $\left(m, n=1,3,5, \ldots, 199 ; T_{0}=C_{0}=0\right)$

\begin{tabular}{lllrrl}
\hline$(\ell / a)^{2}$ & & $\bar{w}$ & \multicolumn{1}{l}{$\bar{\sigma}_{x x}$} & \multicolumn{1}{l}{$\bar{\sigma}_{y y}$} & \multicolumn{1}{l}{$\bar{\sigma}_{x y}$} \\
\hline 0 & $(0)$ & 1.4872 & -2.3277 & -2.0522 & 4.9225 \\
& $(0 / 90)$ & 2.0394 & -3.2269 & 3.2269 & 4.1321 \\
& $(0 / 90 / 0)$ & 1.5543 & -3.2032 & -1.8347 & 4.1702 \\
& $(0 / 90)_{2}$ & 1.6977 & -2.9068 & 2.9068 & 3.2801 \\
0.05 & $(0)$ & 0.8365 & 3.2990 & -1.9125 & 0.5779 \\
& $(0 / 90)$ & 1.0760 & 2.8083 & -2.8083 & 0.6409 \\
& $(0 / 90 / 0)$ & 0.8493 & 3.1336 & -1.8627 & 0.5484 \\
& $(0 / 90)_{2}$ & 0.8919 & 3.3367 & -3.3367 & 0.5251 \\
0.10 & $(0)$ & 0.5652 & 6.3510 & -2.0117 & 0.3675 \\
& $(0 / 90)$ & 0.7226 & 6.0106 & -6.0106 & 0.4153 \\
& $(0 / 90 / 0)$ & 0.5722 & 6.2603 & -1.9841 & 0.3517 \\
& $(0 / 90)_{2}$ & 0.5986 & 6.3938 & -6.3938 & 0.3408 \\
\hline
\end{tabular}

The results for comparison with references found in the literature were obtained considering a sinusoidal thermal load that varies linearly along the plate thickness $\Delta T(x, y, z)=z T_{1} / h$.

Considering the effective disposition of the plies in terms of thermal properties of the material, it is possible to compare the values obtained with the values reported in the book [55] (Table 1) and in the article [58] (Table 2).

If the effective disposition of the plies is not considered for thermal properties, the comparison with Zenkour (Tables 3, 4) can be carried out.

The validity of the code is demonstrated for sinusoidal loads and without nonlocal parameters. Subsequently, the analysis of the cross- and angle-ply laminates for different values of the ratio of nonlocality and different lamination schemes is discussed.
Fig. 10 Displacements $(\bar{w})$ of cross-ply nanoplates $(0 / 90)_{2}$ (a) and $(0 / 90)_{2}$ (b) subjected to uniform hygrothermal load, for different values of $a / b$ ratio and nonlocal parameter $(\ell / a)^{2}$

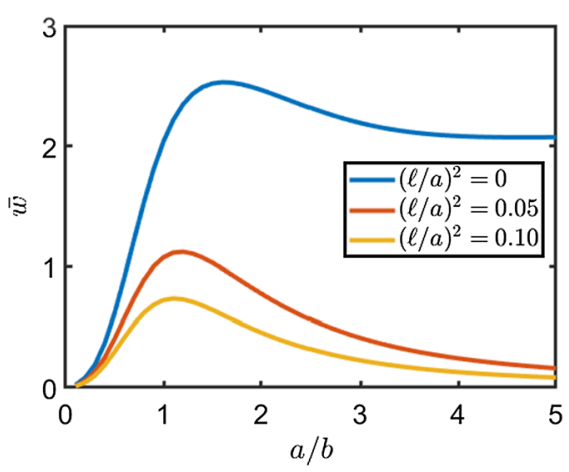

(a)

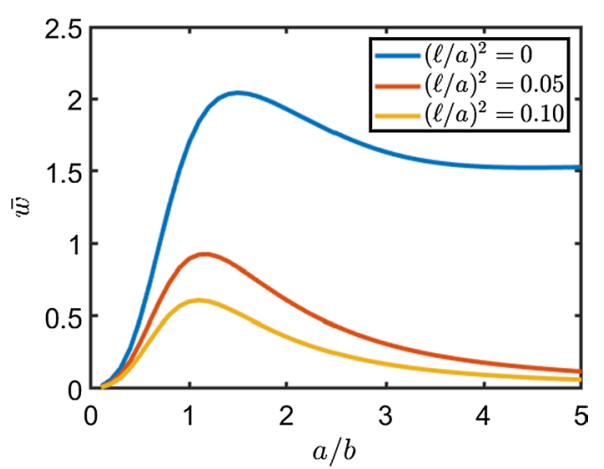

(b) 
Fig. 11 Stresses $(\bar{\sigma})$ of square plates $(0 / 90)(\mathbf{a}-\mathbf{c})$ and $(0 / 90)_{2}$ (d-f) subjected to uniform hygrothermal load, for different nonlocal parameter $(\ell / a)^{2}$

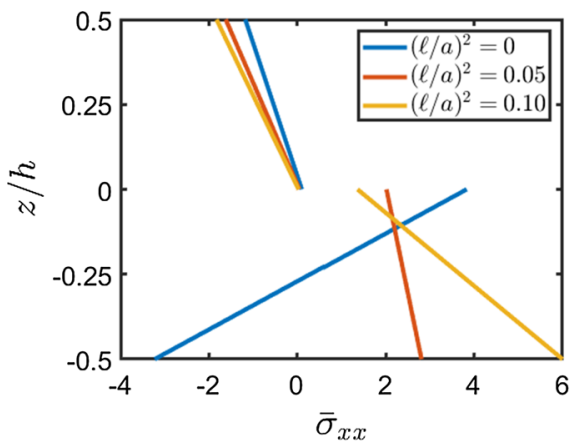

(a)

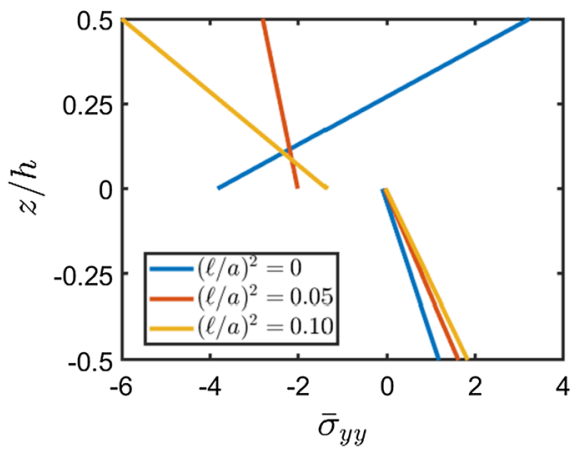

(b)

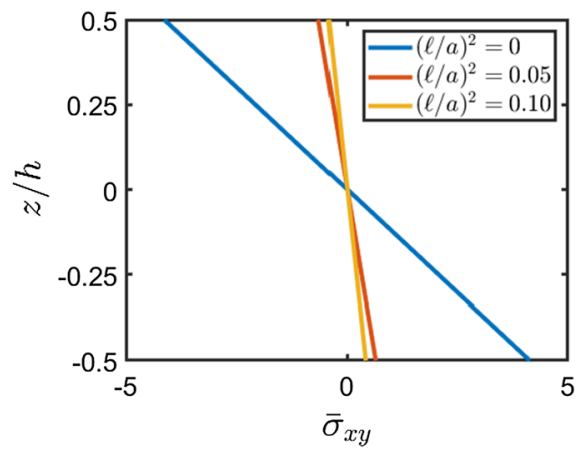

(c)

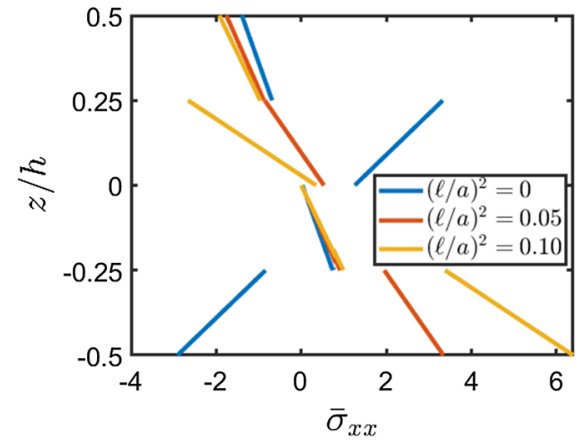

(d)

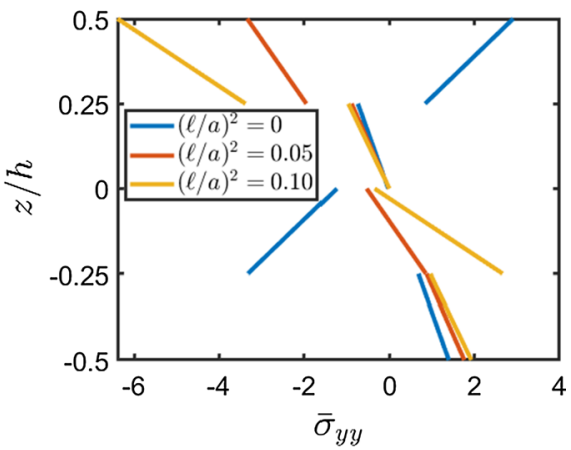

(e)

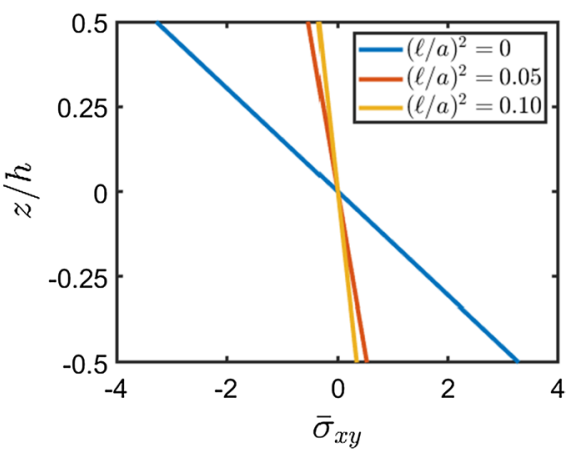

(f)

\subsection{Cross-ply laminates}

In Table 5, the results obtained for a sinusoidal thermal load with linear distribution along the plate thickness are shown. It is noted that the symmetrical laminates have the same behavior independently from the nonlocal parameter, while a significant reduction of displacements and an increase of normal stresses is observed as the ratio $(\ell / a)^{2}$ increases, for the tangential stress there is instead a decrease.

In Fig. 2, the behavior of the antisymmetric plates, when the ratio between $a$ and $b$ sides and the nonlocal parameter vary, is analyzed. From the graphs, it is noted how the vertical displacement stabilizes after reaching the ratio $a / b=1.5$ and also the reduction of the vertical displacement as the nonlocal parameter increases.

Figure 3 represents the normal stresses in the two directions and the tangential in-plane stress, of the plate subjected to the sinusoidal thermal load with linear distribution along the thickness. The plates considered are squared with constant $a / h$ ratio and composed by two and four crossed laminae, respectively. From these graphs, it can be observed how the normal stresses and the shear stress have different trends, 
Table 9 Displacements $(\bar{w})$ and stresses $(\bar{\sigma})$ of a simply supported square angle-ply nanoplates, with different layout and for different values of nonlocal parameter, subjected to a sinusoidal thermal load distribution $\left(T_{0}=C_{0}=0\right)$

\begin{tabular}{llllll}
\hline$(\ell / a)^{2}$ & & $\bar{w}$ & $\bar{\sigma}_{x x}$ & $\bar{\sigma}_{y y}$ & $\bar{\sigma}_{x y}$ \\
\hline 0 & $(-45 / 45)$ & 0.7246 & 3.2024 & -3.2024 & 3.8865 \\
& $(-45 / 45)_{2}$ & 0.5822 & 3.5988 & -3.5988 & 3.5253 \\
& $(-45 / 45)_{4}$ & 0.5688 & 3.6039 & -3.6039 & 3.5235 \\
0.05 & $(-45 / 45)$ & 0.3647 & 5.2220 & -5.2220 & 1.9560 \\
& $(-45 / 45)_{2}$ & 0.2930 & 5.4215 & -5.4215 & 1.7742 \\
& $(-45 / 45)_{4}$ & 0.2863 & 5.4240 & -5.4240 & 1.7733 \\
0.10 & $(-45 / 45)$ & 0.2437 & 5.9010 & -5.9010 & 1.3069 \\
& $(-45 / 45)_{2}$ & 0.1958 & 6.0343 & -6.0343 & 1.1854 \\
& $(-45 / 45)_{4}$ & 0.1913 & 6.0360 & -6.0360 & 1.1848 \\
\hline
\end{tabular}

when the nonlocal parameter increases the first ones registering an increase while the second ones show a decrease.

In order to study the uniform temperature distribution, it was necessary first to study the convergence of the solution by increase $m$ and $n$ because, unlike the sinusoidal distribution that represents a closed form solution, a sufficient number of trigonometric functions are needed to accurately approximate the load. Figure 4 shows in double logarithmic scale the relative error with respect to the expansion order used.

The convergence analysis shows that $m, n=199$ is an excellent approximation for $\bar{w}$ and an acceptable solution in terms of $\bar{\sigma}$, so this value was used in the following applications.

The vertical displacements of the plate subject to uniform thermal load (Table 6) are greater than the previous case.

In Fig. 5, the displacements as a function of the $a / b$ ratio are shown, where in particular you can see the peak of the displacements for the ratio that assumes a value between 1.5 and 2 after which it undergoes a slight flexion and tends to stabilize, whereas with the nonlocal parameter other than zero, the vertical displacements after the peak do not stabilize but continue to decrease with increasing aspect ratios.

As for the sinusoidal thermal load, also for the uniform one there are the increase of normal stresses and the decrease of shear stress, these effects are well visible in Fig. 6.

Once the part related only to the thermal load has been completed, the combined load is analyzed, i.e., a distribution of temperature and a concentration of humidity acting simultaneously on the cross laminated plate. The values of the two loads acting on the plate are $T_{0}=0, T_{1}=100$ and $C_{0}=0, C_{1}=3 \times 10^{-4}$ and are both distributed linearly along the plate thickness (Fig. 7).

In Fig. 8, the trend of normal and shear stresses is shown, along the thickness of two laminates that differ from each other for the number of plies, both subjected to hygrothermal load.

As aforementioned, to study the uniform distribution it is necessary to perform the convergence analysis as shown in Fig. 9. This analysis is carried out both on the relative error on the displacements and on the stresses. It is underlined that the stresses result to have much lower precision in comparison to the displacements, for which a not large trigonometric expansion would be needed to obtain an accurate result.

In Fig. 10, according to what previously detected for the uniform load, the peak of the displacements around $a / b=1.5$ and after a slight bending that tends to stabilize for the higher values of the $a / b$ is observed.

Finally in Fig. 11, the plots of normal and tangential stresses along the thickness of the laminates, with layout $(0 / 90)$ and $(0 / 90)_{2}$, subjected to uniform hygrothermal load and for different values of the nonlocal parameter, are reported results are listed in Tables 7 and 8 .

\subsection{Angle-ply laminates}

No values could be found in the literature for the antisymmetric angle-ply plates in order to carry out a comparison
Fig. 12 Displacements $(\bar{w})$ of angle-ply nanoplates (-45/45) (a) and $(-45 / 45)_{2}$ (b) subjected to sinusoidal thermal load, for different values of $a / b$ ratio and nonlocal parameter $(\ell / a)^{2}$

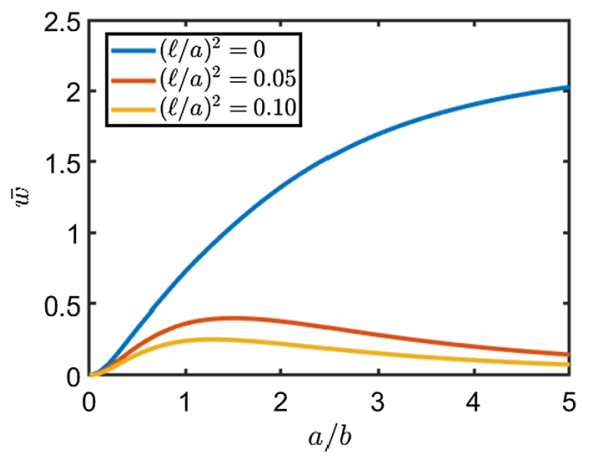

(a)

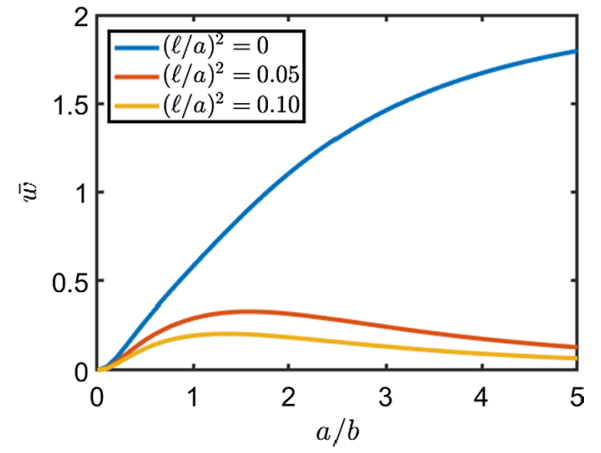

(b) 
Fig. 13 Stresses $(\bar{\sigma})$ of square plates $(-45 / 45)(\mathbf{a}-\mathbf{c})$ and $(-45 / 45)_{2}$ (d-f) subjected to sinusoidal thermal load, for different nonlocal parameters $(\ell / a)^{2}$

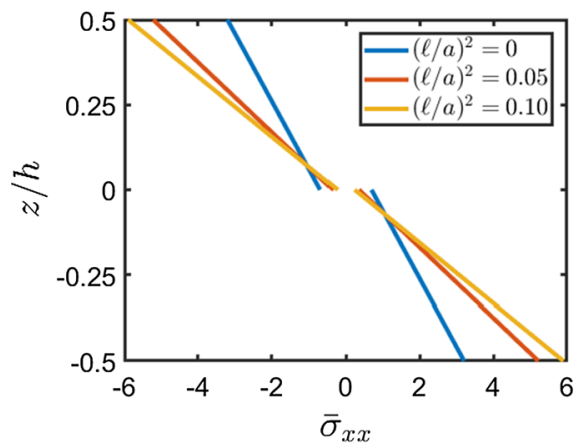

(a)

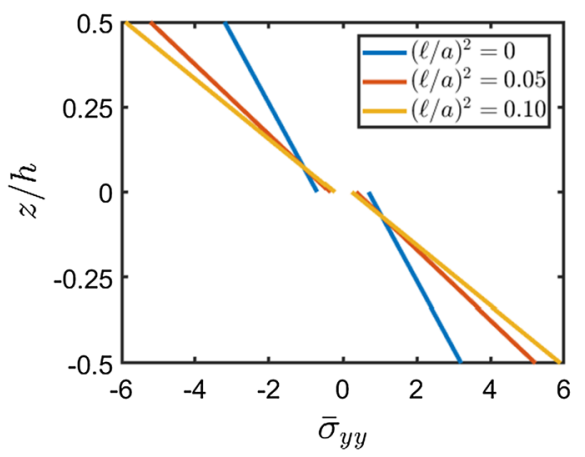

(b)

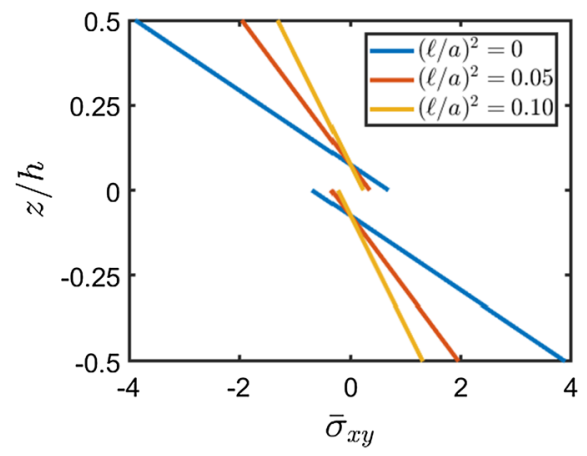

(c)

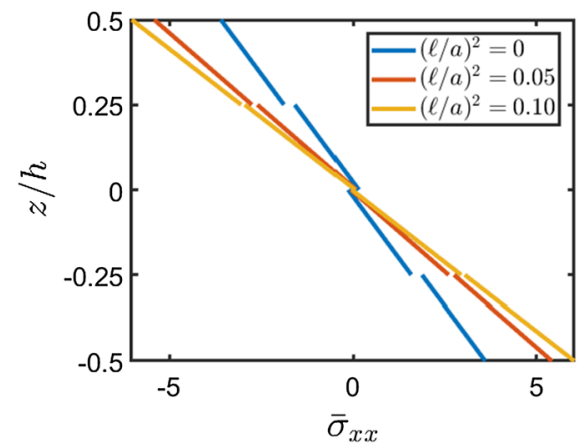

(d)

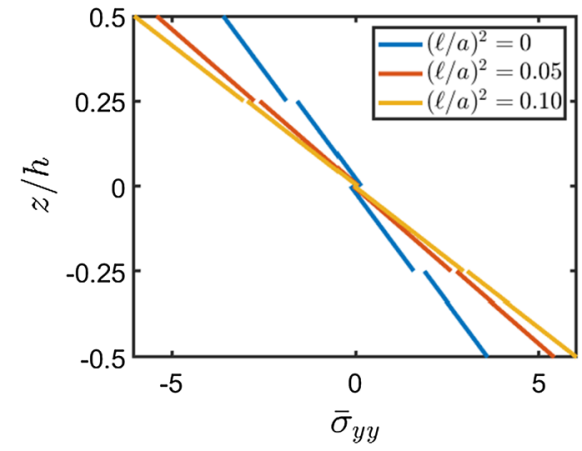

(e)

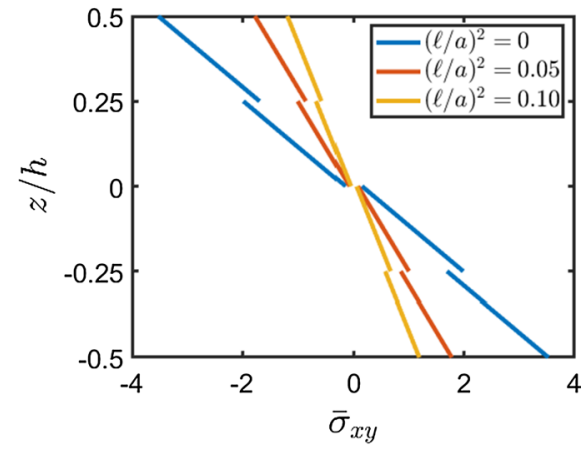

(f)

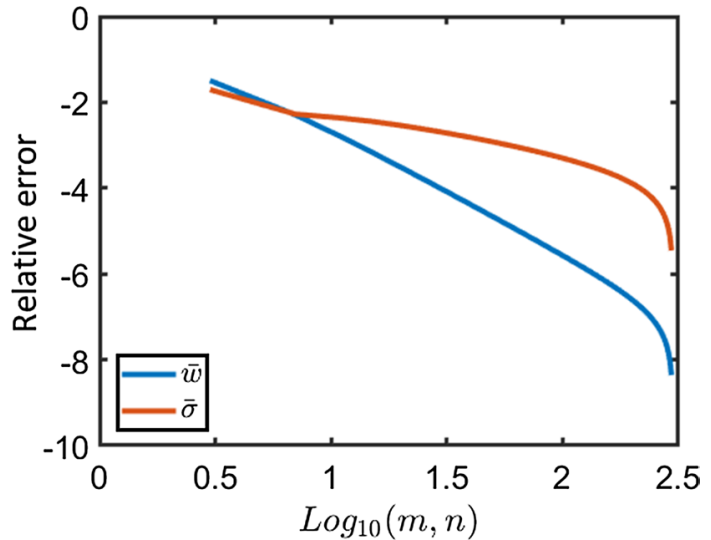

Fig. 14 Relative error in logarithmic scale by varying of $n, m$ for uniform thermal load as previously done for cross-ply laminated plates. Except for the comparison with the literature values, the cases studied follow similar cases as in the previous section. The material properties also remain unchanged compared to the case of cross laminated plates.

It is analyzed the behavior of several angle-ply square plates subjected to a sinusoidal thermal load $\left(T_{0}=0, T_{1}=1\right)$ distributed linearly along the thickness. The results, for the different layout and nonlocal parameter values, are presented in Table 9 and in Fig. 12.

Figure 13 depicts the normal and shear stresses for angleply nanoplates subjected to sinusoidal thermal load. 
Fig. 15 Displacements $(\bar{w})$ of angle-ply nanoplates $(-45 / 45)$ (a) and $(-45 / 45)_{2}$ (b) subjected to uniform thermal load, for different values of $a / b$ ratio and nonlocal parameter $(\ell / a)^{2}$

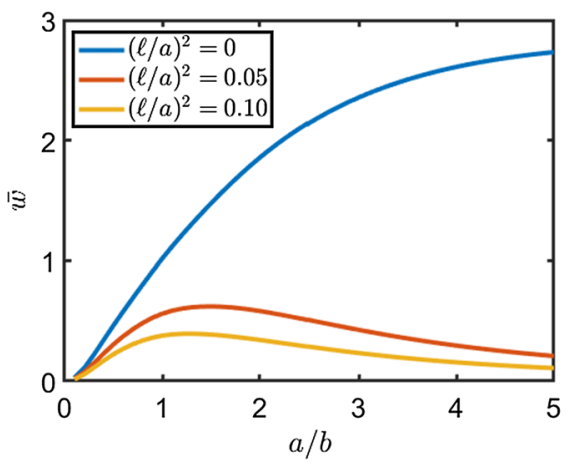

(a)

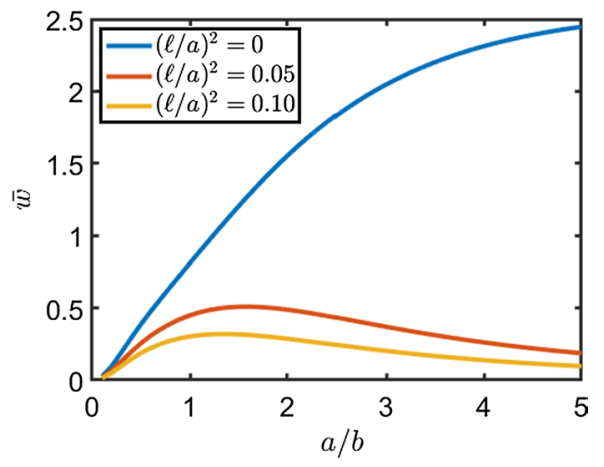

(b)
Table 10 Displacements $(\bar{w})$ and stresses $(\bar{\sigma})$ of a simply supported square angle-ply nanoplate, with different layout and for different values of nonlocal parameter, subjected to a uniform thermal load distribution $(m, n=1,3,5, \ldots, 199$; $\left.T_{0}=C_{0}=0\right)$

\begin{tabular}{llllll}
\hline$(\ell / a)^{2}$ & & $\bar{w}$ & $\bar{\sigma}_{x x}$ & $\bar{\sigma}_{y y}$ & $\bar{\sigma}_{x y}$ \\
\hline 0 & $(-45 / 45)$ & 1.0184 & 4.5920 & -4.5920 & 28.0253 \\
& $(-45 / 45)_{2}$ & 0.8093 & 4.7182 & -4.7182 & 29.4244 \\
& $(-45 / 45)_{4}$ & 0.7897 & 4.5746 & -4.5746 & 31.3414 \\
0.05 & $(-45 / 45)$ & 0.5612 & 4.7544 & -4.7544 & 3.8661 \\
& $(-45 / 45)_{2}$ & 0.4491 & 4.9801 & -4.9801 & 3.6439 \\
& $(-45 / 45)_{4}$ & 0.4386 & 4.9590 & -4.9590 & 3.7088 \\
0.10 & $(-45 / 45)$ & 0.3785 & 5.4754 & -5.4754 & 2.4890 \\
& $(-45 / 45)_{2}$ & 0.3031 & 5.6379 & -5.6379 & 2.3310 \\
& $(-45 / 45)_{4}$ & 0.2961 & 5.6267 & -5.6267 & 2.3655 \\
\hline
\end{tabular}

To study the uniform temperature distribution, it was necessary to carry out a convergence analysis of the results, as it was done in the previous section. Figure 14 displays in double logarithmic scale the relative error as function of the trigonometric expansion considered. As for the case of the cross-ply nanoplates also here $m, n=199$ is considered sufficient (as also indicated by Reddy [12] for elastic plates) for a good approximation of the results.

Once again an increase is observed in the vertical displacement of the plate under the action of a uniform load compared to the sinusoidal thermal load (Fig. 15 and Table 10).
In Fig. 16, normal and shear in-plane stresses are shown along the thickness of the laminates, with layout $(-45 / 45)$ and $(-45 / 45)_{2}$, subjected to uniform thermal load and for different values of the nonlocal parameter.

In the following, the case of plates subjected to both thermal load and hygrometric concentration is studied. The results of a sinusoidal distribution of the loads will be reported first (Figs. 17, 18 and Table 11) and then those related to the uniform distribution (Figs. 20, 21 and Table 12) with relative convergence analysis (Fig. 19). The material properties remain those already used for cross laminated plates. 
Fig. 16 Stresses $(\bar{\sigma})$ of square plates $(-45 / 45)(\mathbf{a}-\mathbf{c})$ and $(-45 / 45)_{2}(\mathbf{d}-\mathbf{f})$ subjected to uniform thermal load, for different nonlocal parameter $(\ell / a)^{2}$

Fig. 17 Displacements $(\bar{w})$ of angle-ply nanoplates $(-45 / 45)$ (a) and $(-45 / 45)_{2}$ (b) subjected to sinusoidal hygrothermal load, for different values of $a / b$ ratio and nonlocal parameter $(\ell / a)^{2}$

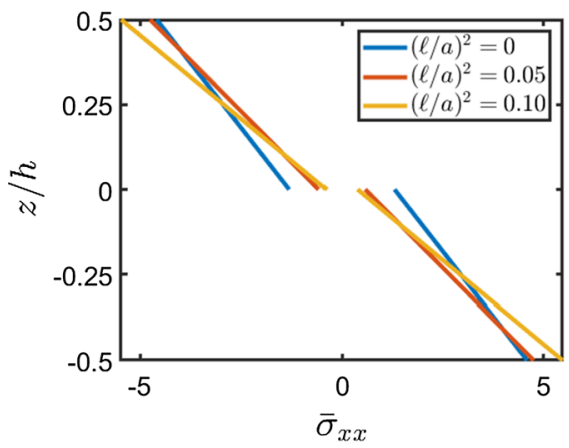

(a)

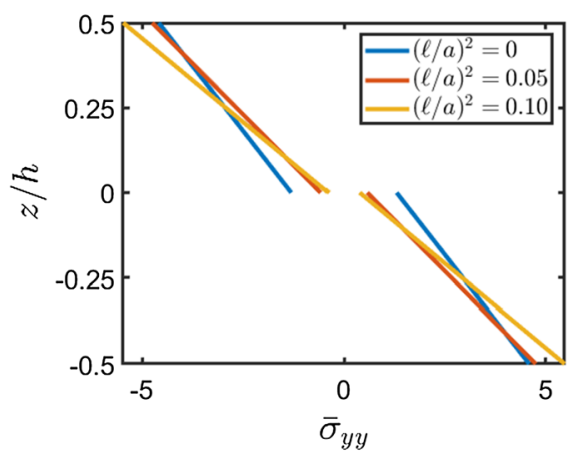

(b)

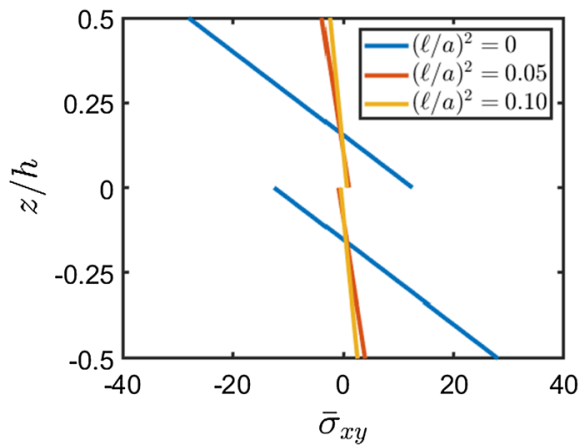

(c)

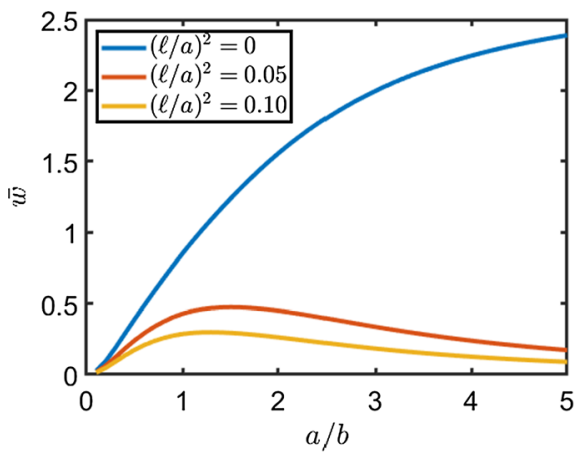

(a)

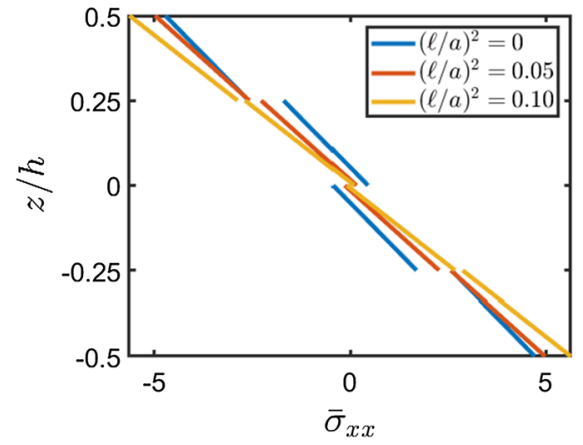

(d)

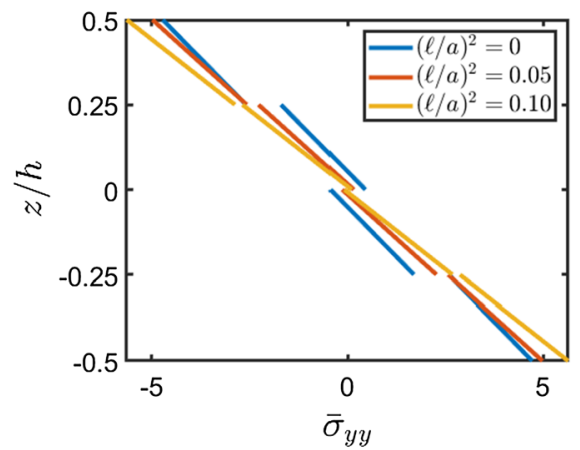

(e)

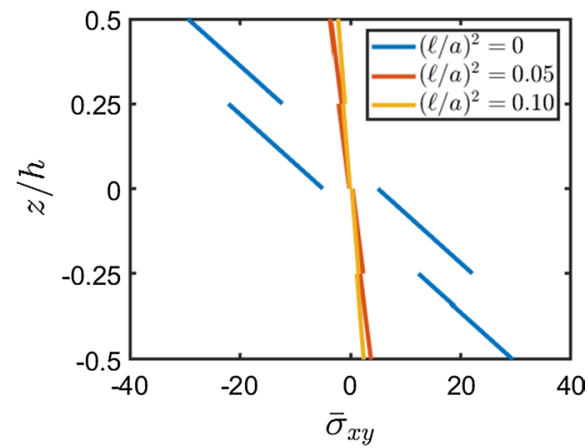

(f)

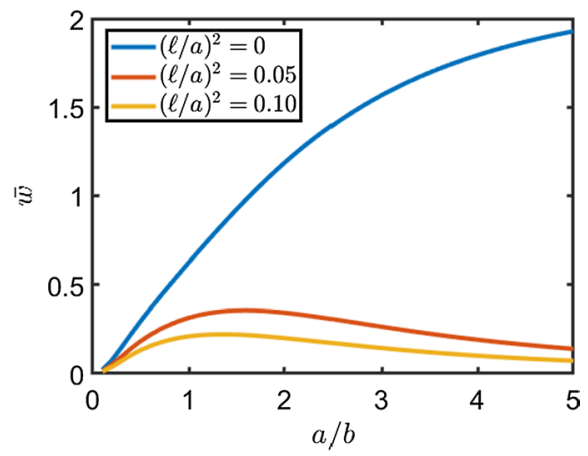

(b) 
Fig. 18 Stresses $(\bar{\sigma})$ of square plates $(-45 / 45)(\mathbf{a}-\mathbf{c})$ and $(-45 / 45)_{2}(\mathbf{d}-\mathbf{f})$ subjected to sinusoidal hygrothermal load, for different nonlocal parameter $(\ell / a)^{2}$

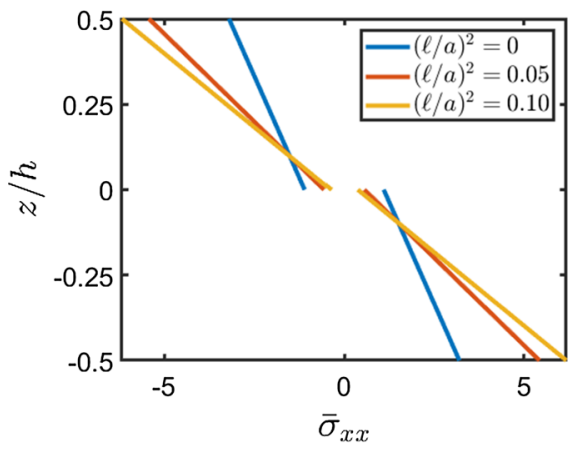

(a)

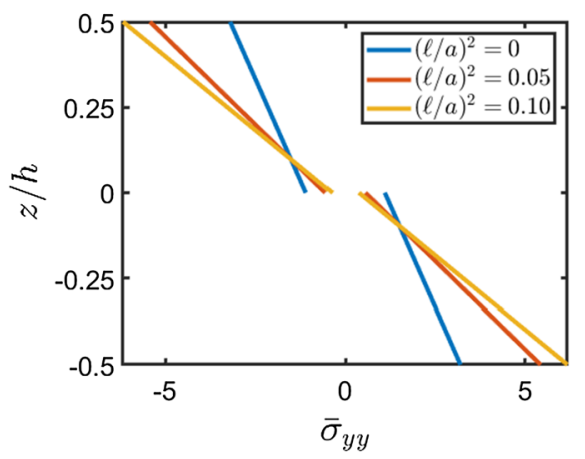

(b)

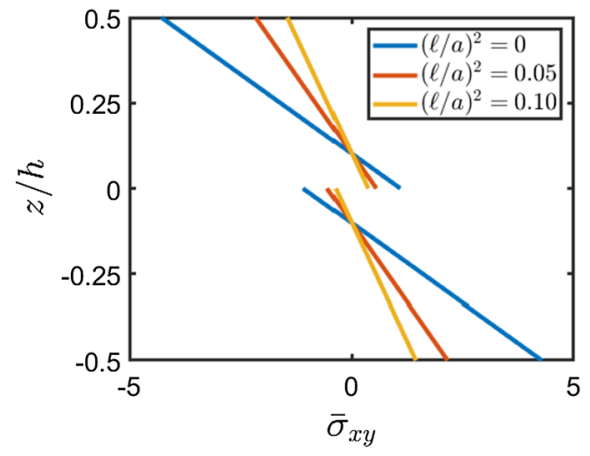

(c)

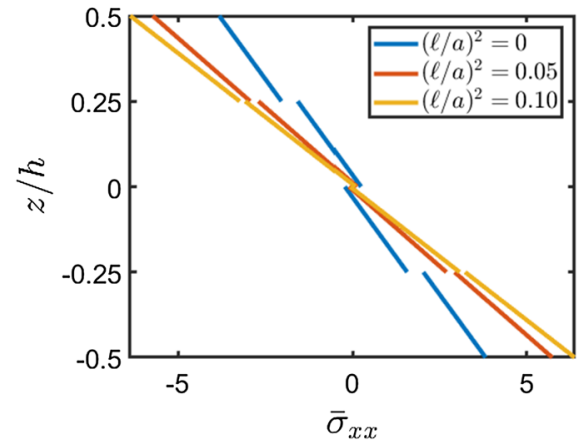

(d)

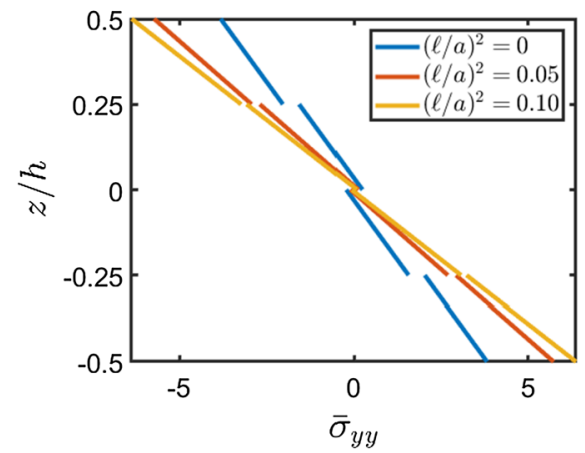

(e)

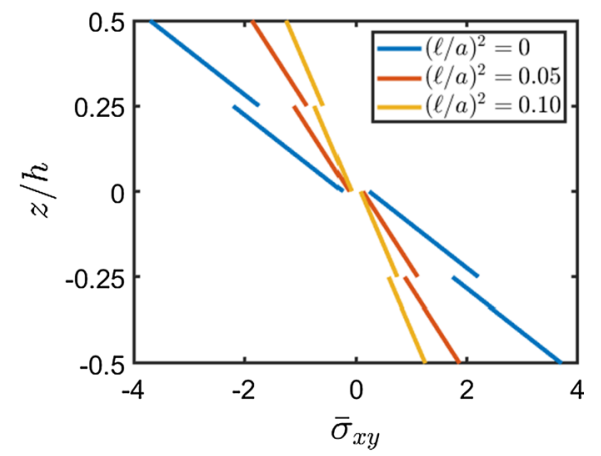

(f)

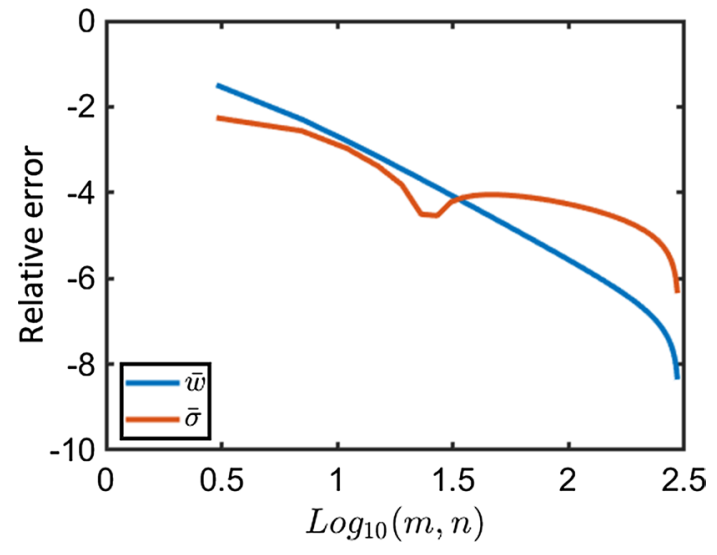

Fig. 19 Relative error in logarithmic scale by varying of $n, m$ for uniform hygrothermal load

\section{Conclusions}

This paper investigates the bending behavior of simply supported cross-ply and angle-ply nanoplates subjected to hygrothermal load using nonlocal strain gradient theory in combination with Kirchhoff plate theory. The analytical solution is obtained thanks to Navier displacement fields. Outcomes have been compared to other works wherever possible, showing good agreement. In the work, an increase in stiffness was observed after the introduction of the nonlocal parameter $\ell$.

Many results are presented here for the first time. For sinusoidal distribution, the thermal and hygrothermal problems are developed for both cross- and angle-ply laminates, and for uniform distribution in addition to displacements and 
Fig. 20 Displacements $(\bar{w})$ of angle-ply nanoplates $(-45 / 45)$ (a) and $(-45 / 45)_{2}$ (b) subjected to uniform hygrothermal load, for different values of $a / b$ ratio and nonlocal parameter $(\ell / a)^{2}$

Fig. 21 Stresses $(\bar{\sigma})$ of square plates $(-45 / 45)(\mathbf{a}-\mathbf{c})$ and $(-45 / 45)_{2}(\mathbf{d}-\mathbf{f})$ subjected to uniform hygrothermal load, for different nonlocal parameter $(\ell / a)^{2}$

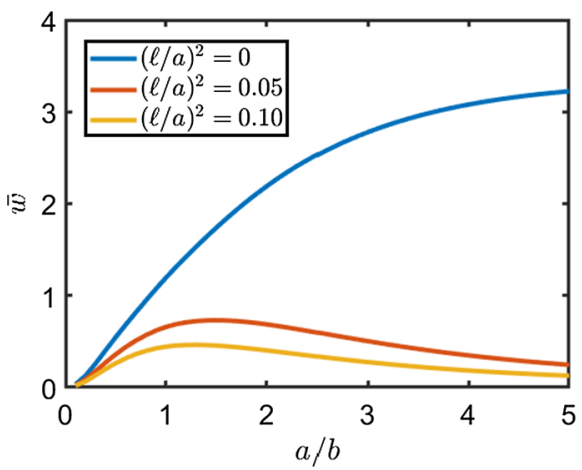

(a)

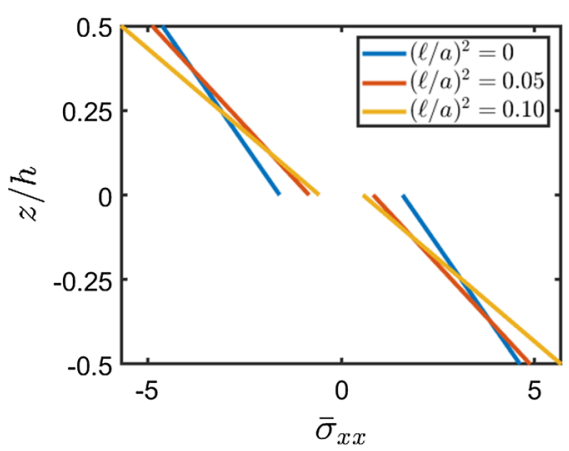

(a)

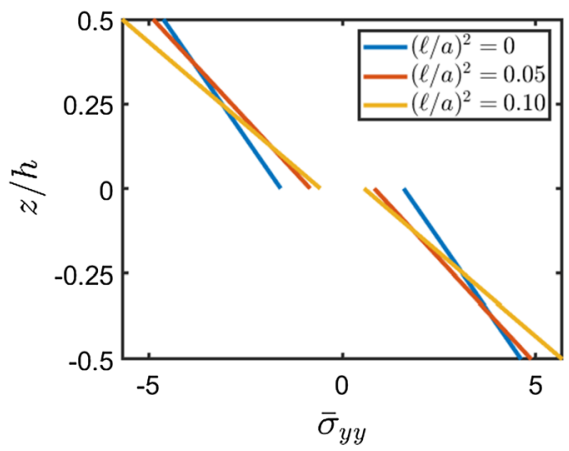

(b)

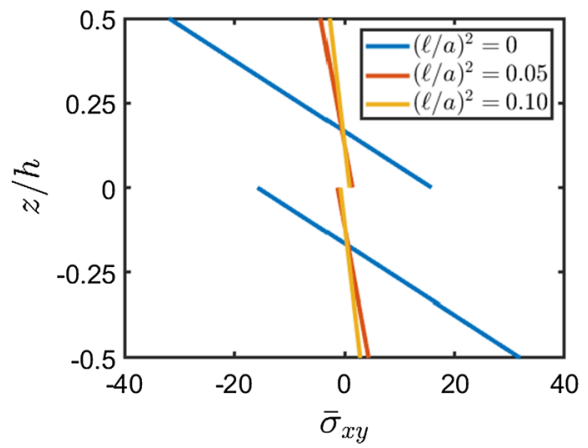

(c)

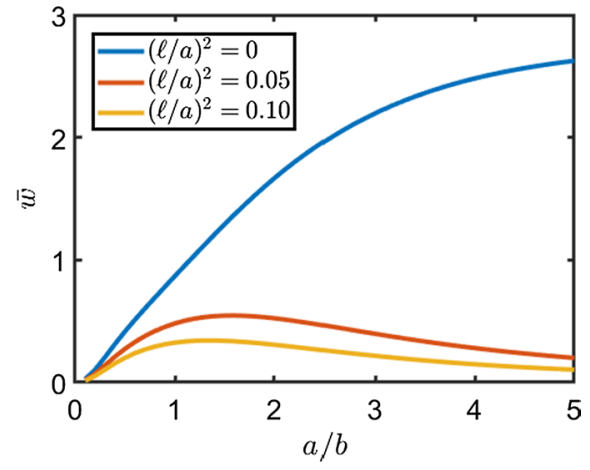

(b)

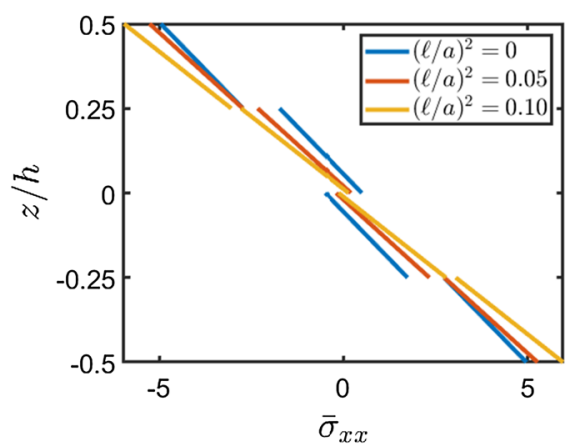

(d)

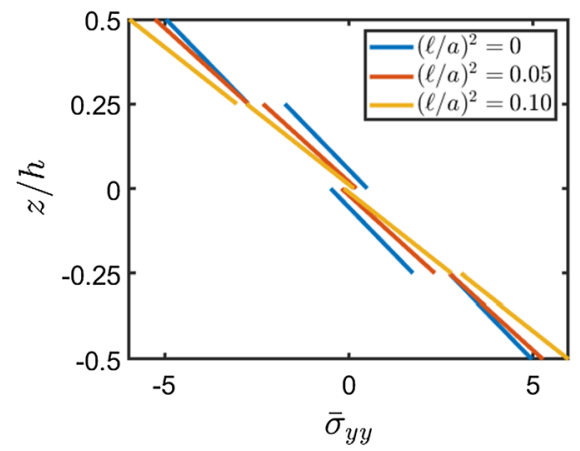

(e)

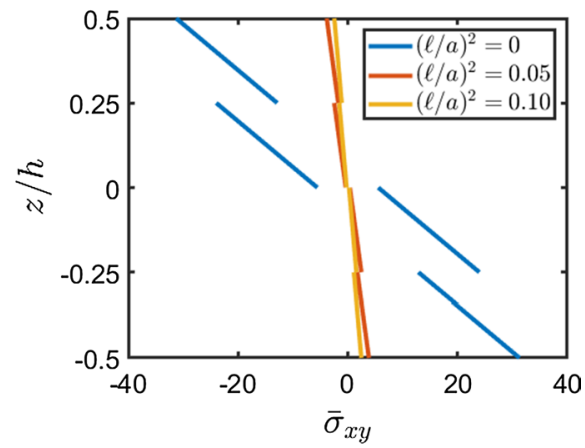

(f) 
Table 11 Displacements $(\bar{w})$ and stresses $(\bar{\sigma})$ of a simply supported square angle-ply nanoplates, with different layout and for different values of nonlocal parameter, subjected to a sinusoidal hygrothermal load distribution $\left(T_{0}=C_{0}=0\right)$

\begin{tabular}{llllll}
\hline$(\ell / a)^{2}$ & & $\bar{w}$ & $\bar{\sigma}_{x x}$ & $\bar{\sigma}_{y y}$ & $\bar{\sigma}_{x y}$ \\
\hline 0 & $(-45 / 45)$ & 0.8507 & 3.1923 & -3.1923 & 4.2790 \\
& $(-45 / 45)_{2}$ & 0.6244 & 3.8220 & -3.8220 & 3.7053 \\
& $(-45 / 45)_{4}$ & 0.6032 & 3.8301 & -3.8301 & 3.7024 \\
0.05 & $(-45 / 45)$ & 0.4281 & 5.4222 & -5.4222 & 2.1536 \\
& $(-45 / 45)_{2}$ & 0.3143 & 5.7392 & -5.7392 & 1.8648 \\
& $(-45 / 45)_{4}$ & 0.3036 & 5.7433 & -5.7433 & 1.8634 \\
0.10 & $(-45 / 45)$ & 0.2860 & 6.1721 & -6.1721 & 1.4389 \\
& $(-45 / 45)_{2}$ & 0.2100 & 6.3838 & -6.3838 & 1.2459 \\
& $(-45 / 45)_{4}$ & 0.2028 & 6.3866 & -6.3866 & 1.2450 \\
\hline
\end{tabular}

Table 12 Displacements $(\bar{w})$ and stresses $(\bar{\sigma})$ of a simply supported square angle-ply nanoplates, with different layout and for different values of nonlocal parameter, subjected to a uniform hygrothermal load distribution $\left(m, n=1,3,5, \ldots, 199 ; T_{0}=C_{0}=0\right)$

\begin{tabular}{llllll}
\hline$(\ell / a)^{2}$ & & $\bar{w}$ & $\bar{\sigma}_{x x}$ & $\bar{\sigma}_{y y}$ & $\bar{\sigma}_{x y}$ \\
\hline 0 & $(-45 / 45)$ & 1.1948 & 4.6252 & -4.6252 & 31.8959 \\
& $(-45 / 45)_{2}$ & 0.8679 & 4.9666 & -4.9666 & 31.2768 \\
0.05 & $(-45 / 45)_{4}$ & 0.8374 & 4.8339 & -4.8339 & 33.1066 \\
& $(-45 / 45)$ & 0.6587 & 4.8896 & -4.8896 & 4.2928 \\
& $(-45 / 45)_{2}$ & 0.4817 & 5.2693 & -5.2693 & 3.8420 \\
0.10 & $(-45 / 45)_{4}$ & 0.4651 & 5.2513 & -5.2513 & 3.9031 \\
& $(-45 / 45)$ & 0.4443 & 5.6941 & -5.6941 & 2.7598 \\
& $(-45 / 45)_{2}$ & 0.3251 & 5.9635 & -5.9635 & 2.4565 \\
& $(-45 / 45)_{4}$ & 0.3140 & 5.9545 & -5.9545 & 2.4889 \\
\hline
\end{tabular}

stresses outcomes, the convergence analysis is carried out. These results can be used as benchmark for further studies within the same topic.

Funding Open access funding provided by Alma Mater Studiorum Università di Bologna within the CRUI-CARE Agreement.

Open Access This article is licensed under a Creative Commons Attribution 4.0 International License, which permits use, sharing, adaptation, distribution and reproduction in any medium or format, as long as you give appropriate credit to the original author(s) and the source, provide a link to the Creative Commons licence, and indicate if changes were made. The images or other third party material in this article are included in the article's Creative Commons licence, unless indicated otherwise in a credit line to the material. If material is not included in the article's Creative Commons licence and your intended use is not permitted by statutory regulation or exceeds the permitted use, you will need to obtain permission directly from the copyright holder. To view a copy of this licence, visit http://creativecommons.org/licenses/by/4.0/.

\section{References}

1. Saji VS, Choe HC, Yeung KW (2010) Nanotechnology in biomedical applications: a review. Int J Nano Biomater 3:119-139

2. Berman D, Krim J (2013) Surface science, MEMS and NEMS: progress and opportunities for surface science research performed on, or by, microdevices. Prog Surf Sci 88:171-211

3. Bhushan B (2007) Nanotribology and nanomechanics of MEMS/ NEMS and bioMEMS/bioNEMS materials and devices. Microelectron Eng 84:387-412

4. Ekinci KL, Roukes ML (2005) Nanoelectromechanical systems. Rev Sci Instrum 76:061101

5. Bonanni A, del Valle M (2010) Use of nanomaterials for impedimetric DNA sensors: a review. Anal Chim Acta 678:7-17

6. Wu W (2017) Inorganic nanomaterials for printed electronics: a review. Nanoscale 9:7342-7372

7. Gohardani O, Elola MC, Elizetxea C (2014) Potential and prospective implementation of carbon nanotubes on next generation aircraft and space vehicles: a review of current and expected applications in aerospace sciences. Prog Aerosp Sci 70:42-68

8. Singh T (2014) A review of nanomaterials in civil engineering works. Int J Struct Civ Eng Res 3:31-5

9. Fabbrocino F, Carpentieri G (2017) Three-dimensional modeling of the wave dynamics of tensegrity lattices. Compos Struct 173:9-16

10. Mancusi G, Fabbrocino F, Feo L, Fraternali F (2017) Size effect and dynamic properties of 2D lattice materials. Compos B Eng 112:235-242

11. Tarantino AM (2008) Homogeneous equilibrium configurations of a hyperelastic compressible cube under equitriaxial dead-load tractions. J Elast 92:227

12. Reddy J (2007) Theory and analysis of elastic plates and shells. CRC Press, Berlin

13. McFarland AW, Colton JS (2005) Role of material microstructure in plate stiffness with relevance to microcantilever sensors. J Micromech Microeng 15:1060-1067

14. Lam D, Yang F, Chong A, Wang J, Tong P (2003) Experiments and theory in strain gradient elasticity. J Mech Phys Solids 51:1477-1508

15. Barretta R, Fabbrocino F, Luciano R, de Sciarra FM, Ruta G (2020) Buckling loads of nano-beams in stress-driven nonlocal elasticity. Mech Adv Mater Struct 27:869-875

16. Lakes R (1986) Experimental microelasticity of two porous solids. Int J Solids Struct 22:55-63

17. Stölken J, Evans A (1998) A microbend test method for measuring the plasticity length scale. Acta Mater 46:5109-5115

18. Tuna M, Kirca M, Trovalusci P (2019) Deformation of atomic models and their equivalent continuum counterparts using Eringen's two-phase local/nonlocal model. Mech Res Commun 97:26-32

19. Kai M, Zhang L, Liew K (2020) Carbon nanotube-geopolymer nanocomposites: a molecular dynamics study of the influence of interfacial chemical bonding upon the structural and mechanical properties. Carbon 161:772-783

20. Izadi R, Tuna M, Trovalusci P, Ghavanloo E (2021) Torsional characteristics of carbon nanotubes: Micropolar elasticity models and molecular dynamics simulation. Nanomaterials 11:453

21. Trovalusci P (2014) Molecular approaches for multifield continua: origins and current developments, pp 211-278

22. Eringen AC (1983) On differential equations of nonlocal elasticity and solutions of screw dislocation and surface waves. J Appl Phys $54: 4703-4710$

23. Aifantis E (2003) Update on a class of gradient theories. Mech Mater 35:259-280 
24. Meenen J, Altenbach H, Eremeyev V, Naumenko K (2011) A variationally consistent derivation of microcontinuum theories. Adv Struct Mater 15:571-584

25. Yang F, Chong A, Lam D, Tong P (2002) Couple stress based strain gradient theory for elasticity. Int J Solids Struct 39:2731-2743

26. Mühlhaus H, Oka F (1996) Dispersion and wave propagation in discrete and continuous models for granular materials. Int J Solids Struct 33:2841-2858

27. Leonetti L, Greco F, Trovalusci P, Luciano R, Masiani R (2018) A multiscale damage analysis of periodic composites using a couplestress/Cauchy multidomain model: application to masonry structures. Compos B Eng 141:50-59

28. Trovalusci P, Bellis MD, Ostoja-Starzewski M (2016) A statistically-based homogenization approach for particle random composites as micropolar continua. Adv Struct Mater 42:425-441

29. Reccia E, De Bellis ML, Trovalusci P, Masiani R (2018) Sensitivity to material contrast in homogenization of random particle composites as micropolar continua. Compos B Eng 136:39-45

30. Fantuzzi N, Leonetti L, Trovalusci P, Tornabene F (2018) Some novel numerical applications of Cosserat continua. Int J Comput Methods 15:1850054

31. Mindlin R, Eshel N (1968) On first strain-gradient theories in linear elasticity. Int J Solids Struct 4:109-124

32. Barretta R, Feo L, Luciano R, Marotti de Sciarra F, Penna R (2016) Functionally graded Timoshenko nanobeams: a novel nonlocal gradient formulation. Compos B Eng 100:208-219

33. Karami B, Janghorban M, Rabczuk T (2019) Static analysis of functionally graded anisotropic nanoplates using nonlocal strain gradient theory. Compos Struct 227:111249

34. Eremeyev V, Altenbach H (2015) On the direct approach in the theory of second gradient plates. Shell Mem Theor Mech Biol 45:147-154

35. Bacciocchi M, Fantuzzi N, Ferreira A (2020) Conforming and nonconforming laminated finite element Kirchhoff nanoplates in bending using strain gradient theory. Comput Struct 239:106322

36. Sahmani S, Aghdam MM, Rabczuk T (2018) Nonlinear bending of functionally graded porous micro/nano-beams reinforced with graphene platelets based upon nonlocal strain gradient theory. Compos Struct 186:68-78

37. Jamalpoor A, Hosseini M (2015) Biaxial buckling analysis of double-orthotropic microplate-systems including in-plane magnetic field based on strain gradient theory. Compos B Eng 75:53-64

38. Papargyri Beskou (2008) Static, stability and dynamic analysis of gradient elastic flexural Kirchhoff plates. Arch Appl Mech 97:625-635

39. Cornacchia F, Fantuzzi N, Luciano R, Penna R (2019) Solution for cross- and angle-ply laminated Kirchhoff nano plates in bending using strain gradient theory. Compos B Eng 173:107006

40. Togun N, Bagdatli SM (2016) Nonlinear vibration of a nanobeam on a Pasternak elastic foundation based on non-local Euler-Bernoulli beam theory. Math Comput Appl 21:3

41. Akgoz B, Civalek O (2015) Bending analysis of FG microbeams resting on Winkler elastic foundation via strain gradient elasticity. Compos Struct 134:294-301

42. Civalek O, Demir C, Akgoz B (2010) Free vibration and bending analyses of cantilever microtubules based on nonlocal continuum model. Math Comput Appl 15:289-298

43. Brischetto S, Leetsch R, Carrera E, Wallmersperger T, Kröplin B (2008) Thermo-mechanical bending of functionally graded plates. J Therm Stresses 31:286-308
44. Brischetto S (2012) Hygrothermal loading effects in bending analysis of multilayered composite plates. Comput Model Eng Sci 88:367-418

45. Brischetto S, Carrera E (2012) Coupled thermo-electro-mechanical analysis of smart plates embedding composite and piezoelectric layers. J Therm Stresses 35:766-804

46. Brischetto S, Carrera E (2013) Static analysis of multilayered smart shells subjected to mechanical, thermal and electrical loads. Meccanica 48:1263-1287

47. Mohammadimehr M, Salemi M, Rousta Navi B (2016) Bending, buckling, and free vibration analysis of MSGT microcomposite Reddy plate reinforced by FG-SWCNTs with temperature-dependent material properties under hydro-thermo-mechanical loadings using DQM. Compos Struct 138:361-380

48. Arefi M, Zenkour AM (2017) Thermo-electro-mechanical bending behavior of sandwich nanoplate integrated with piezoelectric face-sheets based on trigonometric plate theory. Compos Struct 162:108-122

49. Yan J, Tong L, Li C, Zhu Y, Wang Z (2015) Exact solutions of bending deflections for nano-beams and nano-plates based on nonlocal elasticity theory. Compos Struct 125:304-313

50. Thai CH, Ferreira A, Phung-Van P (2020) A nonlocal strain gradient isogeometric model for free vibration and bending analyses of functionally graded plates. Compos Struct 251:112634

51. Tocci Monaco G, Fantuzzi N, Fabbrocino F, Luciano R (2021) Critical temperatures for vibrations and buckling of magneto-electro-elastic nonlocal strain gradient plates. Nanomaterials 11:87

52. Tocci Monaco G, Fantuzzi N, Fabbrocino F, Luciano R (2021) Trigonometric solution for the bending analysis of magneto-electro-elastic strain gradient nonlocal nanoplates in hygro-thermal environment. Mathematics 9:567

53. Cornacchia F, Fabbrocino F, Fantuzzi N, Luciano R, Penna R (2019) Analytical solution of cross- and angle-ply nano plates with strain gradient theory for linear vibrations and buckling. Mech Adv Mater Struct 2019:1-15

54. Babu B, Patel B (2019) A new computationally efficient finite element formulation for nanoplates using second-order strain gradient Kirchhoff's plate theory. Compos B Eng 168:302-311

55. Reddy J (2003) Mechanics of laminated composite plates and shells: theory and analysis, 2nd edn. CRC Press, Berlin

56. Zenkour A (2004) Analytical solution for bending of cross-ply laminated plates under thermo-mechanical loading. Compos Struct 65:367-379

57. Zenkour A, Allam M, Radwan A (2014) Effects of hygrothermal conditions on cross-ply laminated plates resting on elastic foundations. Arch Civ Mech Eng 14:144-159

58. Naik NS, Sayyad AS (2020) Analysis of laminated plates subjected to mechanical and hygrothermal environmental loads using fifth-order shear and normal deformation theory. Int J Appl Mech 12:2050028

59. Bacciocchi M, Fantuzzi N, Ferreira AJM (2020) Static finite element analysis of thin laminated strain gradient nanoplates in hygro-thermal environment. Continuum Mech Thermodyn 2020:1-24

Publisher's Note Springer Nature remains neutral with regard to jurisdictional claims in published maps and institutional affiliations. 